\title{
MAP4K4 determines cancer cell phenotype by controlling the plasma membrane-associated proteome
}

Charles Capdeville ${ }^{1}$, Linda Russo ${ }^{1}$, David Penton ${ }^{2}$, Jessica Migliavacca ${ }^{1}$, Milica Zecevic ${ }^{1}$, Alexandre Gries $^{1}$, Stephan C.F. Neuhauss ${ }^{2}$, Michael Grotzer ${ }^{3}$, Martin Baumgartner ${ }^{1 *}$

1. Pediatric Molecular Neuro-Oncology Lab, Children's Research Center, University Children's Hospital Zürich, Zürich, Switzerland

2. Department of Molecular Life Sciences, University of Zurich, Zürich, Switzerland

3. Department of Oncology, University Children's Hospital Zürich, Zürich, Switzerland

*Correspondence: Pediatric Molecular Neuro-Oncology Lab, Children's Research Center, University Children's Hospital Zürich, Balgrist Campus, Lengghalde 5, CH-8008 Zürich, Switzerland

CH-8008 Zürich, e-mail: Martin.Baumgartner@kispi.uzh.ch; Tel.: +4144266 3730

\begin{abstract}
How the phenotype of Medulloblastoma (MB) tumor cells adapts in response to growth factor cues is poorly understood. We systematically determined alterations in the plasma membrane (PM)-associated proteome in growth factor-activated MB cells. We found that ligand-induced activation of c-MET receptor tyrosine kinase triggers specific internalization of C-MET and of membrane-associated and transmembrane proteins including nucleoside and ion transporters. In contrast, c-MET activation caused increased PM association of the PVR/CD155 adhesion and immunomodulatory receptor, promoting MB cell motility and tumor cell growth in the cerebellar tissue. Both increased and decreased PM association of a number of these proteins including PVR/CD155 is regulated by the Ser/Thr MAP4K4. We further identified Endophilin A proteins as potential regulators of this process downstream of MAP4K4 to contribute to HGF-induced invasion control. Together, our findings indicate a novel link between MAP4K4 overexpression in MB and the maintenance of a cellular phenotype associated with growth and invasiveness.
\end{abstract}




\section{Introduction}

The plasma membrane (PM) proteome is a dynamic interface of mammalian cells that regulates cellular interactions with the extracellular environment. This physical barrier regulates the transfer of chemical and mechanical information between extracellular and intracellular spaces. The net outcome of the PM barrier functions affects the cellular phenotype and the composition and biophysical structure of the extracellular microenvironment. The PM proteome primarily consists of receptors, cell adhesion molecules, enzymes and ion channels ${ }^{1}$, which define strength and duration of signal transmission and how the cell interacts with its environment. The diversity of proteins associated with the PM and their differential expression, localization or regulation in pathological conditions such as cancers or inflammation not only represents condition-specific biomarkers ${ }^{2}$, but also a druggable repertoire of therapeutic targets to interfere with the oncogenic phenotype.

The PM proteome of a cell is dynamically controlled through membrane turnover via endocytic uptake and exocytic fusion ${ }^{3,4}$. This membrane trafficking is a key mechanism that determines cellular responses to complex environments and enables the uptake of nutrients or extrusion of signal transmitters. In addition, endocytosis and the downstream vesicular trafficking inside the cells control adhesion turnover, transduction, and the duration of mitogenic or mobilizing signals. De-regulation of physiological trafficking in tumor cells may thus contribute to cell transformation ${ }^{5}$, and altered endocytic activity can broadly impact tumor cell growth, invasion and metastasis and viability ${ }^{6,7}$.

Medulloblastoma (MB) is a malignant embryonal neuroepithelial tumor of the cerebellum with a high propensity to disseminate and metastasize within the central nervous system ${ }^{8}$. Several omics studies described the genomic heterogeneity in MB, which is now classified into four subgroups with a total of twelve subtypes ${ }^{9,10}$. Despite this molecular understanding of MB, the current standard therapy still consists of surgical resection of the tumor and craniospinal irradiation followed by chemotherapy ${ }^{11}$. These anti-cancer therapies can have a devastating impact on the developing brain of the patients and can cause severe long-term side effects such as cognitive or mobility impairments ${ }^{12}$. Thus, novel therapeutic options with phenotype-specific anti-tumor activity are needed to replace or complement current treatments and reduce therapy-associated side effects.

The Ser/Thr kinase MAP4K4 is overexpressed in MB and contributes to the pro-invasive phenotype of MB cells downstream of hepatocyte growth factor (HGF)-cellular mesenchymal-epithelial transition factor (c-MET) stimulation ${ }^{13,14}$. c-MET receptor tyrosine kinase (RTK) activation caused increased membrane dynamics, increased endocytic uptake and the activation and turnover of integrin ß1 adhesion receptor $^{13}$. Integrin activation and internalization of integrins are necessary for cell migration and invasion ${ }^{15,16}$. Internalization of activated RTKs and integrins furthermore modulates pathway activation at endosomal compartments ${ }^{17,18}$, thereby adding a layer of regulatory impact of endocytic activity on tumor cell behavior. How the PM proteome of MB cells is regulated in response to RTK activation and potential therapeutic targets in the dynamic PM compartment are not known.

Endophilins are members of the BAR (Bin/amphiphysin/Rvs) domain superfamily that play an essential role in cellular processes like synaptic vesicle endocytosis, receptor trafficking, apoptosis and mitochondrial network dynamics ${ }^{19,20}$. Dysregulation of these processes are associated with cancer or 
neurodegenerative diseases ${ }^{19,21}$. Endophilins were originally considered a peripheral component of clathrin-mediated endocytosis 22,23 . A novel clathrin-independent endocytic pathway based on endophilin A subfamily activity, termed fast endophilin mediated endocytosis (FEME) was described recently ${ }^{21}$, and several studies also implicated endophilins in cell motility control ${ }^{24-26}$. The endophilin A1 is almost exclusively expressed in brain tissue ${ }^{27}$

Herein we used a spatial proteomics approach ${ }^{28}$ and determined the PM-associated proteome of resting and RTK-activated MB cells. We furthermore addressed whether the pro-oncogenic activity of MAP4K4 could be coupled to RTK-induced, dynamic alterations in the PM proteome. This analysis allowed the identification of PM-associated proteins subjected to c-MET-induced spatial re-distribution and the identification of MAP4K4 as a driver of this process.

\section{Results}

\section{HGF-C-MET signaling promotes PM proteome reorganization in medulloblastoma cells}

To determine changes in PM and PM-proximal association of proteins in response to growth factor stimulation, we established a PM proteome analysis approach for MB cells. We used membraneimpermeable Sulfo-NHS-SS-biotin labeling of intact sgControl (sgCTL, gC) and sgMAP4K4 (MAP4K4 knockout, gM4) MB tumor cells, followed by streptavidin protein capture and high-resolution mass spectrometry analysis (Fig. 1A). To determine whether PM association of protein expression in MB cells is regulated by growth factor receptor activation, we compared starved and HGF-stimulated cells. Proteins identified were filtered either using the transmembrane (TM) prediction algorithm $\mathrm{TMHMM}^{29}$ or by subcellular localization annotation ${ }^{30}$. These stringent sorting criteria identified 952 TM proteins and 336 PM-associated proteins, respectively (Tables 1 \& 2). In sgCTL cells, HGF stimulation significantly decreased the abundance of a number of TM (Fig. 1B, Table 3) or PM-annotated proteins (Fig. 1C, Table 4). This indicated that either internalization of these proteins in response to c-MET activation is increased, or recycling decreased. We previously found that internalization of activated c-MET depends on MAP4K4 ${ }^{13}$. Therefore, we tested whether MAP4K4 is required for reduced PM association in cells, where c-MET is activated by HGF stimulation. Depletion of MAP4K4 (Fig. S1A) induced increased detection of TM (Fig. 1B, Table 3) or PM-associated proteins (Fig. 1C, Table 4) after c-MET activation, suggesting a regulatory function of MAP4K4 in PM localization of these proteins. A paradigmatic example is c-MET itself, which is internalized in a MAP4K4-dependent manner in DAOY cells ${ }^{14}$ and detected at lower levels in the PM proteome in response to HGF ligation (Fig. 1D). MAP4K4 is necessary for this process as depletion of MAP4K4 significantly increases c-MET abundance in HGFstimulated cells (Fig. 1D). None of the five additional RTKs detected by mass-spectrometry displayed similar behavior, suggesting that RTK internalization either requires the precedent activation of the receptor kinase domain or is specific for c-MET. We concluded from this experiment that c-MET activation triggers the turnover of PM-associated proteins and that MAP4K4 is indispensable for the turnover of a number of those proteins. Gene ontology analysis of TM-associated proteins revealed MAP4K4 dependencies in several pathways controlling endo- and exocytotic activities of cells as well as immune cell recognition or solute exchange (Fig. 1E). It also revealed that MAP4K4 depletion in HGF-stimulated cells increased PM association of proteins with reported implication in chemotherapy 
sensitivity/resistance (SLC29A1, SLC4A7, LRRC8) ${ }^{31,32}$. Conversely, MAP4K4 depletion decreased PM association of proteins involved in tumor immune evasion (CD155, CD276, HLA-G) ${ }^{33-35}$, in solute influx regulation (CLIC1, SLCs, ABCCs) ) $^{36,37}$ and cell migration (CD155, CD276) $)^{33,38}$ (Fig. 1F).

Taken together, our data suggested that MAP4K4 is involved in mediating HGF-C-MET induced turnover of PM-associated proteins. However, MAP4K4 also enables the maintenance of some transporters and receptors such as CLIC1, CD155, and CD276 at the PM after MET activation (Fig. 1F). In conclusion, we found that MET activation by HGF triggers reorganization of the PM-associated proteome in MB cells and that MAP4K4 selectively controls this turnover.

\section{Depletion of MAP4K4 moderately decreases sensitivity to Lomustine}

Membrane transporters and channels are proteins involved in the transfer of ions, nutrients and drugs across the PM. Dysregulation of influx or efflux mechanisms can lead to both chemotherapy sensitivity or resistance in cancer cells ${ }^{39,40}$. We detected altered PM association of transporters including SLC29A1, SLC4A7, ABCC1, ABCB1 and LRRC8A (Fig. S2A), where contribution to the cytotoxic drug influx/efflux has been proposed. SLC29A1 for example mediates the uptake of Lomustine (CCNU) and Azacytidine ${ }^{31}$ and SLC4A7 controls uptake of Mitomycin and Spiromustine ${ }^{31}$. ABCB1 mediates the efflux of Etoposide and Bisantrene ${ }^{31,41}$ and ABCC1 of Methotrexate and Irinotecan ${ }^{42}$. LRRC8A is a bi-directional transporter and mediates influx/efflux of drugs such as Cisplatin and Temozolomide ${ }^{32,43}$. In our experimental settings, HGF stimulation reduced PM association of SLC29A1, SLC4A7 and LRRC8A in sgCTL cells (Fig. S2A). Reduced PM association depends on MAP4K4 as plasma membrane association of these transporters is increased in sgMAP4K4 cells stimulated with HGF (Fig. S2A). MAP4K4 depletion also causes a significant increase in PM-association of ABCB1 and ABCC1 in HGF-stimulated cells (Fig. S2A). These differences in PM association are not the consequences of altered regulation of gene expression (Fig. S1B). Thus, these results suggested a potential implication of HGF stimulation and MAP4K4 expression in the influx/efflux of cytotoxic drugs. To test this possibility, we generated dose-response curves for Lomustine ${ }^{44}$ and Etoposide ${ }^{45}$ in DAOY cells. Under normal growth conditions (10\%FBS), depletion of MAP4K4 did not significantly impact the sensitivity of DAOY cells to Etoposide compared to control cells (IC50 value of $3.2 \mu \mathrm{M}$ and $2.8 \mu \mathrm{M}$ respectively) but slightly increased resistance to Lomustine (IC50 value of $46.2 \mu \mathrm{M}$ and $39.5 \mu \mathrm{M}$ respectively) (Fig. S2B). To investigate the effect of HGF stimulation on drug response, cells were first maintained in low serum media for $24 \mathrm{~h}$ (1\% FBS) and then treated for $48 \mathrm{~h}$ with HGF in combination with Lomustine or Etoposide. Under these conditions, depletion of MAP4K4 moderately reduced Lomustine sensitivity (Fig. S2C). We observed no impact on the response to Etoposide treatment. HGF stimulation did not affect the sensitivity of the cells to the two drugs, independent of the MAP4K4 expression level. Taken together, these results suggest a moderately sensitizing effect of MAP4K4 on tumor cell sensitivity to Lomustine.

\section{MAP4K4 controls ion homeostasis and modulates PM localization of potassium channels}

To better understand which protein functions are particularly affected by HGF stimulation and/or MAP4K4 depletion, we annotated the filtered proteins using Protein Atlas ${ }^{46,47}$. We found that 223 of 952 
transmembrane proteins (Fig. S3A) and 84 of 336 plasma membrane proteins (Fig. S3B) are annotated as transporters. These trans-membrane proteins mediate the transfer of ions, small molecules, and macromolecules across membranes ${ }^{48}$, thus suggesting the potential implication of the HGF-c-METMAP4K4 axis in MB ion homeostasis.

Specifically, HGF-dependent alterations in PM association of ion channels, transporters and exchangers (Fig. S3C), including CLIC1 ( $\mathrm{Cl}^{-}$channel), CLCN7 $\left(\mathrm{Cl}^{-}\right.$channel), SLC12A7 $\left(\mathrm{K}^{+} / \mathrm{Cl}^{-}\right.$ transporter), ATP1B3 ( $\mathrm{Na}+/ \mathrm{K}+$ exchanger) and TMEM165 $\left(\mathrm{H}^{+} / \mathrm{Ca}^{+} / \mathrm{Mn}^{+}\right.$exchanger $)$depends on MAP4K4 expression (Fig. 2A). Depletion of MAP4K4 in HGF-stimulated cells either increases or decreases their PM association compared to HGF-treated control cells (Fig. 2A and S3C). Additionally, pathway enrichment analysis performed on the significantly regulated transmembrane predicted proteins between HGF-stimulated sgMAP4K4 KO cells and control cells revealed an enrichment of TM proteins belonging to the gene ontology for potassium ion homeostasis (Fig. 2B). These proteins include several solute carriers (SLCs) and ATPases. HGF stimulation of sgCTL cells reduced the abundance of these proteins at the PM (Fig. 2C). Depletion of MAP4K4 maintained PM association of most of these SLCs and ATPases in HGF-stimulated cells or resulted even in increased PM association compared to unstimulated cells (Fig. 2C).

To access the functional significance of the HGF- and MAP4K4-dependent modulation of PM localization of ion channels on MB cell ion homeostasis, we performed whole-cell patch clamp measurement of the electrical properties of DAOY cells with or without MAP4K4 expression. In starved conditions, MAP4K4 depletion lead to an increased whole-cell current compared to control cells (Fig. 2D). Quantification of the resting membrane potential $\left(V_{\text {mem }}\right)$ indicated that HGF stimulation forced the hyperpolarization of MAP4K4-depleted cells compared to control cells (Fig. 2E, filled circles). This last finding is in accordance with the hypothesis that disseminating cancer cells present a more depolarized $V_{\text {mem }}$ and that their hyperpolarization leads to loss of their metastatic potential ${ }^{49}$.

One channel with a significantly altered PM association is the chloride intracellular channel protein 1 (CLIC1). CLIC1 contributes to MB proliferation in vitro, and CLIC1 depletion in MB tumor cell lines significantly reduces tumor cell growth in vivo ${ }^{37}$. Gene expression analysis of several MB patient sample cohorts shows CLIC1 overexpression in the tumor samples compared to control tissues (Fig. 2F). Importantly, this elevated CLIC1 expression is associated with worse outcomes in $\mathrm{SHH}$-alpha and Gr4 MB tumors (Fig. 2G). In control cells, PM association of CLIC1 is moderately but not significantly increased by HGF stimulation. In contrast, HGF stimulation of MAP4K4 depleted cells caused a significant drop of CLIC1 PM association (Fig 2A), suggesting that MAP4K4 is either necessary for transporting CLIC1 to the plasma membrane or for CLIC1 maintenance at the PM in growth factorstimulated cells.

Taken together, these data indicated the implication of MAP4K4 in the control of electrophysiological parameters of MB cells. However, the relevance on cell migration or tumor progression remains to be investigated.

\section{HGF-controlled CD155 expression promotes medulloblastoma cell invasion and motility}


We found differential PM association of the adhesion and immune-modulatory proteins CD155 and CD276 (Fig. 1F). CD276 impacts colorectal cancer cell migration through the Jak2/Stat3/MMP-9 signaling pathway ${ }^{38}$. CD155 interacts with integrins or RTKs and increases FAK, Ras, or RAP1 downstream signaling, leading to increased cell motile capabilities ${ }^{50}$. CD155, also referred to as poliovirus receptor (PVR), is a member of the nectin-like family of proteins. CD155 is involved in cell adhesion, migration, proliferation, or immune regulation ${ }^{33}$, and it is often upregulated in tumors compared to healthy tissues ${ }^{50}$. This renders CD155 particularly interesting in the context of tumor progression, where it could contribute to immune evasion and dissemination.

Our data indicated that the PM association of CD155 is moderately increased in HGFstimulated cells. This increase in PM association requires MAP4K4, as depletion of MAP4K4 in HGFstimulated cells causes a significant reduction of CD155 PM association (Fig. 3A). As we did not measure a significant reduction in CD155 mRNA expression in sgMAP4K4 cells, reduced PM association of CD155 in sgMAP4K4 cells stimulated with HGF is not the result of transcriptional repression. Furthermore, HGF stimulation increased mRNA expression of CD155 both in sgCTL and sgMAP4K4 cells by $10-20 \%$ (Fig. 3B). Thus, the significant decrease in CD155 PM association in HGF-stimulated sgMAP4K4 cells is not the consequence of transcriptional repression. To validate MAP4K4 implication in PM association of CD155, we determined CD155 surface expression levels by flow cytometry analysis. HGF stimulation increased surface localization of CD155 in sgCTL cells by 20\% (Fig. 3C). Depletion of MAP4K4 not only prevented increased CD155 surface expression after HGF-stimulation but even caused a $10-20 \%$ reduction of surface-expressed CD155 relative to unstimulated controls cells (Fig. $3 \mathrm{C}$ ). This suggested that HGF triggers increased surface expression of CD155 and that MAP4K4 is either required for the transfer or the maintenance of CD155 at the PM under conditions that trigger membrane dynamics and endocytic turnover.

We hypothesized that reduced PM association of CD155 and CD276 in sgMAP4K4 cells could be causative for the reduced MB cell dissemination observed with these cells ${ }^{13,14}$. To test this hypothesis, we measured the impact of reduced expression of CD155 or CD276 on HGF-induced collagen I invasion. We found that depletion of CD155, but not of CD276, prevented HGF-induced collagen I invasion (Fig. 3D). To corroborate the implication of CD155 in migration control with another cell line, we tested the effect of CD155 depletion on collagen I invasion in ONS-76 cells. These cells invade collagen I independently of exogenous growth factor stimulation. Depletion of CD155 significantly reduced collagen I invasion of ONS-76 cells (Fig. 3E). The reduced invasion could be a consequence of reduced speed of migration. To test this possibility, we performed single-cell motility assays with ONS-76 cells seeded in collagen I coated plates. Cell movements were recorded by timelapse video microscopy for five hours. CD155 depletion reduced the average speed of ONS-76 cells by $50 \%$ (Fig 3F). Phenotypically, cells with decreased CD155 expression displayed increased circularity, increased area and increased width to length ratio (Fig $3 F$, movies 1 and 2).

Taken together, these data identified the CD155 cell adhesion molecules as a promotor of cell motility and invasiveness in SHH MB cell models.

\section{CD155 is necessary for ex vivo cerebellar tissue infiltration}


To assess whether CD155 depletion prevented cerebellar tissue infiltration of MB cells, we implanted ONS-76-LA-EGFP tumor spheroids onto cerebellar slices and determined growth and invasion after tumor-cerebellar slice co-culture ${ }^{51}$. After 48 hours of co-culture, ONS-76-LA-EGFP cells transfected with siCTL displayed sheet-like infiltration of the surrounding brain tissue (Fig. 3G). In contrast, spheroids of cells transfected with siCD155 did not display an invasive behavior and remained clustered at the site of initial spheroid positioning. As an indirect measurand for tumor growth and invasion, we quantified the volume of the tumor cell clusters. We observed a significant ( 2 -fold) reduction of the cluster volume in siCD155 transfected compared to siCTL cells (Fig. 3G, S4). Some cells in the siCD155 condition still display an invasive phenotype, possibly due to variations in transfection efficiency at single-cell level. We also evaluated tumor cell proliferation in the tissueembedded tumor cell clusters using EdU incorporation. We found that depletion of CD155 caused a marked decrease in the number of proliferative cells in the ex vivo model (Fig. 3G, S4).

Together, these findings suggest that the cell adhesion receptor CD155 is critical for effective tissue invasion and growth in SHH MB cells.

\section{Expression of MAP4K4 and of the endocytosis effector EndoA1 is increased in SHH MB}

MAP4K4 is overexpressed in MB patient tumor samples ${ }^{52}$ and gene expression profiling of 763 primary $\mathrm{MB}$ samples ${ }^{53}$ revealed that $M A P 4 K 4$ expression correlates positively with genes involved in endocytosis control (Fig. S5A). Interestingly, the strongest positive correlate with MAP4K4 expression among the endocytosis-regulating genes is SH3GL2 (EndoA1, $r=0.501$ ), while expression of both CLTB $(r=-0.228)$ and CTLC ( $r=-0.228)$ correlate negatively with MAP4K4 (Fig. S5B). The highest positive correlation between SH3GL2 and MAP4K4 expression is observed in SHH MB tumors, where also CLTC and SH3GL2 expression correlate negatively ( $r=-0.144)$ (Fig. S5C). These correlation analyses indicated that the decrease in cell migration observed in MAP4K4-depleted cell ${ }^{52}$ could be mechanistically linked to a clathrin-independent mechanism of endocytosis. Increased levels of MAP4K4, SH3GL2 and SH3GL3 - both at protein and RNA levels - but not of SH3GL1, were also observed in $\mathrm{MB}$ and ganglioglioma samples of a recent genomic and proteomic analysis of a cohort of 218 pediatric brain tumors ${ }^{54}$ (Fig. S5D). These findings revealed high expression of endophilins in MB compared to other central nervous system tumors and indicated the potential importance of fastendophilin-mediated endocytosis $\left(\mathrm{FEME}^{21}\right)$ in this tumor type.

\section{EndoA family proteins are involved in HGF-induced MB cell migration}

To explore endocytic pathways (Fig. S6A) involved in HGF-induced migration control more broadly, we determined investigated which endocytic pathways are necessary for maintaining the HGFinduced invasive phenotype. We used 25 siRNAs targeting 16 key components of 8 different endocytic pathway (Fig. S6B) to identify relevant components. Downregulation efficacy of the siRNAs was confirmed by qRT-PCR (Fig. S6C). The Boyden Chamber transwell migration assay was used to quantify siRNA impact on HGF induced transwell motility (Fig. 4A). Consistent with previous studies ${ }^{13,52}$, HGF stimulation increased the number of transmigrated cells $(\sim 2.7$-fold increase). Depletion of dynamins 1 and 2 (DNM1 and DNM2) abrogated HGF-induced migration (Fig. 4A), confirming a general 
role of endocytosis in migration control. Interestingly, depletion of Endophilin A proteins completely abrogated (SH3GL2, EndoA1) or drastically reduced (SH3GL1, EndoA2 or SH3GL3, EndoA3) HGFinduced cell migration (Fig. 4A). In contrast, depletion of clathrin or caveolin, two essential components of endocytic pathways for GF signaling ${ }^{55}$, did not prevent or reduce HGF-induced DAOY cell migration. Similarly, depletion of two regulators of micropinocytosis, CTBP1 and RAC ${ }^{56}$, did also not block HGFinduced migration. Both non-transfected and siCTL transfected cells showed similar levels of proliferation up to 72 hours, while depletion of SH3GL1/2/3 and DNM1 exhibited even moderately increased levels of proliferation (Fig. S6D). These data exclude a proliferation defect as underlying cause of the smaller number of transmigrated cells counted in the siSH3GL1/2/3- or siDNM1transfected cells and indicate the implication of EndoA1 and DNM1 in migration control.

\section{Endophilin $\mathrm{A} 1$ is required for $3 \mathrm{D}$ collagen I matrix invasion}

We next tested whether depletion of EndoA1, DNM1, or clathrin heavy chain (CLTC) also affects 3D collagen I invasiveness using the spheroid invasion assay (SIA) ${ }^{57,58}$ (Fig. 4B, S6E). HGF caused a robust increase in the number of cells disseminating into the collagen I matrix and increased the distance of invasion ( 6.8-fold increase compared to untreated control). Depletion of EndoA1 or DNM1 significantly reduced HGF-induced collagen I invasion, whereas depletion of CLTC had no effect, indicating that EndoA1 functions in clathrin-independent endocytosis. Consistent with a conserved role of EndoA1 in migration control, depletion of EndoA1 also reduced ( 1.6-fold decrease) collagen I invasion of ONS-76 cells (Fig. 4C). Depletion of MAP4K4 did not affect expression of EndoA1 (Fig. $\mathrm{S} 6 \mathrm{~F}$ ). To determine whether a compensatory mechanism mediated by EndoA2 or EndoA3 ${ }^{24}$ may be active in our cell model, we depleted all Endophilin-A proteins simultaneously (TKD). We found that endophilin TKD abrogates HGF-induced cell migration in DAOY cells moderately more effective than siEndoA1 alone (Fig. 4D). We concluded that EndoA2 or EndoA3 contribute to migration control, similar to what was observed in other cell models ${ }^{24}$, and that EndoA1 is the most relevant Endophilin in our cell model. In conclusion, we found that EndoA1 mediates a migratory phenotype in MB cells, possibly through a mechanism involving clathrin-independent endocytosis.

\section{Endophilin-A1 depletion reduces tissue invasion}

We next assessed the role of EndoA1 in tumor growth and invasion control in the tissue context with tumor-cerebellar slice co-culture experiments using ONS-76-LA-EGFP cells. Depletion of EndoA1 markedly reduced cerebellum tissue infiltration and prevented the formation of streams of invading cells, which are highly characteristic for this type of MB tumor cell (Fig. 4E, panel a). Individual EndoA1depleted tumor cells still displayed invasion at the border of the spheres. However, the morphology of these cells appeared more rounded, less mesenchymal compared to control cells (Fig. 4, panel b). Although morphology of the collectivity of the invading cells varied between samples, the considerably reduced invasiveness of EndoA1-depleted cells was apparent, while proliferation did not seem to be significantly affected in vitro (Fig. S6D) and ex vivo (Figs 4E, S6G). Taken together, these data indicate that EndoA1 contributes to the invasive behavior of the tumor cells in the cerebellar tissue without affecting proliferation. 


\section{MAP4K4 controls subcellular localization of CIP4}

Disturbance of vesicle trafficking is expected when endocytic processes are targeted. We therefore determined whether EndoA1- or MAP4K4-depleted cells display altered subcellular abundance and distribution of early and late endosome markers compared to control cells using antiEEA1 and anti-LAMP1 antibodies, respectively (Fig. S7). Depletion of EndoA1 and MAP4K4 led to a $20 \%$ reduction of EEA1 puncta per cell (Fig. S7A), indicating that both EndoA1 and MAP4K4 enable formation of early endosomes. Depletion of MAP4K4 but not of EndoA1 also reduced the number of LAMP1 puncta per cell by $15 \%$ (Fig. S7B). Thus, in addition to contributing to early steps of endocytosis, MAP4K4 could also be involved in later steps of the endosomal degradation pathway. HGF stimulation for 10 or 30 min did not affect formation of EEA1-positives vesicles but reduced the number of LAMP1positive vesicles after $30 \mathrm{~min}$. This decrease in LAMP1-positive vesicles was not observed in cells depleted of EndoA1 (Fig. S7B), indicating that EndoA1 could re-route vesicles away from the degradation pathway in HGF-stimulated cells.

To test whether MAP4K4 could be involved in PM localization of FEME components, we searched the proteins detected in the surface proteome (Tables 1 \& 2) for CIN85/SH3KBP1, CIP4 and FBP17, three regulators of FEME priming ${ }^{59}$ (Fig. 5A). This experiment suggested that HGF stimulation caused a moderately increased PM association of SH3KBP1/CIN85, CIP4, FNBP1/FBP17 and EndoA2 (Fig. 5B). Increased PM association of these proteins depends on MAP4K4, as PM association under HGF stimulation is abrogated in cells depleted of MAP4K4. EndoA1 and EndoA3 were not detected. CIN85/SH3KBP1, CIP4 and FNBP1/FBP17 are considered critical mediators of FEME by priming the $\mathrm{PM}$ for endophilin A clustering ${ }^{59}$. PM enrichment of these proteins in a MAP4K4-dependent manner in HGF-stimulated cells could indicate a link between MAP4K4 and FEME priming. Interestingly, we also detected the potential interaction of SH3KBP1/CIN85 with MAP4K4 in an unrelated proteomic interaction analysis ${ }^{60}$. Due to their cytoplasmic localization, we further tested whether MAP4K4 depletion alters subcellular localization of CIP4. We found that depletion of EndoA1 and MAP4K4 increased cortical localization of CIP4 in circular patches near cellular protrusions (Fig. 5B). Interestingly, we also observed EEA1 staining in similar patches in siCTL-transfected cells (Fig. S7A). These patches were not apparent in siEndoA1 and siMAP4K4-transfected cells (Fig. S7A). CIP4 is associated with microtubule-like structures ${ }^{61}$, and consistently we observed a filamentous pattern of CIP4 staining that does not co-localize with F-actin (Fig. 5C), a known target of MAP4K4 activity ${ }^{13,52}$.

These results indicate the implication of MAP4K4 in membrane recruitment, cortical localization and organization of key regulators of FEME, thereby possibly facilitating an effective FEME sequence ${ }^{59}$ (Fig. 5D).

\section{Discussion}

We found that HGF-induced c-MET activation caused significant alterations in the abundance of transmembrane and plasma membrane-associated proteins. The abundance of a majority of these proteins is reduced by HGF-c-MET activation, indicating either GF-dependent internalization or attenuated recycling of PM-associated proteins. We found that PM association of some of these 
proteins is MAP4K4 dependent. MAP4K4 control of PVR/CD155 PM association is particularly intriguing as we also found that PVR/CD155 is necessary for efficient tumor cell migration and brain tissue invasion. Our data furthermore point towards a role of fast-endophilin-mediated endocytosis in migration control through the EndoA protein family and provide a conceptual basis for novel strategies to target the oncogenic phenotype of $\mathrm{MB}$ and other solid tumors.

PM-associated proteins were enriched by biotin labeling prior to mass-spectrometry analysis. This methodology is expected to label predominately extracellularly bound or anchored proteins, transmembrane proteins, and proteins associated with endocytic vesicles. To exclude non-PM proteins, we either used the TMHMM algorithm to predict transmembrane domains in proteins ${ }^{29}$ or a data-based filtering approach, yielding two datasets. Former is highly efficient to detect surface proteins. However, it also predicts transmembrane domains of proteins associated with other membrane compartments such as the golgi or ER membranes. The data-based filtering approach selects proteins based on published subcellular localization (detailed annotations available in Table 2). This approach is not specifically enriching for proteins with transmembrane domains, and it may include non-PM proteins complexed with PM-associated proteins. The TMHMM and data-based annotation approach selected $21 \%$ and $8 \%$ of all proteins detected in the initial MS analyses, respectively. The TMHMM approach was highly efficient for determining MAP4K4 impact on PM-association of transporters and channels, which all contain a TM domain. The data-based annotation approach is highly stringent, and it allows for the comparative analysis of the variations in PM association in all the conditions tested using pathway enrichment analysis. Therefore, we used latter for most of the subsequent analyses.

MAP4K4 implication in HGF-induced internalization of membrane transport proteins and drug transporters ${ }^{31}$ (SLC29A1, ABCC1, ABCB1, LRCC8) indicated a potential therapeutic benefit of targeting this function of MAP4K4 during chemotherapy, which remains one of the first line treatments for cancer patients. We hypothesized that indirect modulation of drug up-take or extrusion mechanisms could improve drug efficacy. MAP4K4 depletion moderately decreased the susceptibility of DAOY cells to Lomustine, but it had no effect on etoposide treatment efficacy. Although of potential interest, our data are inconclusive and additional chemotherapeutic drugs and cell lines should be tested to identify specific susceptibilities. Disturbed cell-surface localization of ion channels and transporters could furthermore modify the overall ion homeostasis of the cell. The changes in PM association of ion channels we observed is associated with subtle alterations in electrical currents. This is consistent with previous studies that demonstrated altered electrical conductance properties in tumors compared to healthy tissues ${ }^{37,62,63}$. We identified CLIC1, CLIC4, SLC29A1 or ATP1A3 among others as channels susceptible to HGF or MAP4K4 regulation. Increased current in MAP4K4-depleted cells indicates MAP4K4 repression of ion channel distribution or activity that could control ion homoeostasis of the tumor cells. The lowered potential in MAP4K4-depleted cells after HGF stimulation could be the consequence of a reduced influx of cations, increased efflux of cations or increased influx of anions. Pathway enrichment analysis of PM associated channels pointed towards an altered potassium homeostasis. We observed increased PM-association of potassium channels and potassium transportassociated molecules including the P-type cation transporter ATP12A, the $\mathrm{Ca}^{2+-}$ dependent channels ATP1A3 and KCNMA1 in MAP4K4-depleted cells after HGF stimulation. Thus, MAP4K4 could maintain 
optimal polarization in HGF-stimulated cells by controlling potassium efflux rates through regulating $\mathrm{PM}$-association of $\mathrm{K}+$ transporters. However, whether differential PM association of potassium channels and de-regulated potassium fluxes are at the origin of the electrical disbalance observed in MAP4K4-depleted cells and contribute to the MAP4K4-associated pro-migratory phenotype remains to remains to be determined.

We observed reduced PM-association of CD155 and CD276 in MAP4K4-depleted cells stimulated with HGF. In glioma cells, CD155 mediates adhesion to the extracellular matrix protein vitronectin, promotes FA turnover and FA signaling towards SRC, paxillin and p130CAS, and thereby contributes to tissue invasion ${ }^{64}$. MAP4K4 contributes to FAK activation at the leading edge of migrating cells ${ }^{14}$, and our data herein indicate a possibly indirect regulation of this process via the control of CD155 surface expression. Our observation of a more rounded, less mesenchymal morphology in CD155-depleted cells has been described previously in glioma cells ${ }^{14}$, and studies in triple negative aggressive breast cancer cells demonstrated CD155 contribution to the mesenchymal cell state ${ }^{65}$. Depletion of CD155 in these cells triggered mesenchymal to epithelial transition and repressed migration and invasion in vitro and in vivo., suggesting that CD155 expression in MB cells could also contribute to migration control indirectly via transcriptional reprogramming. CD155 also represents an attractive therapeutic target due to its abilities to inhibit NK cell and CD8 ${ }^{+} \mathrm{T}$-cell activity, thereby contributing to immune evasion of tumor cells ${ }^{66,67}$. Kinase-controlled modulation of CD155 surface expression as we found herein enable this process as rather the abundance - and not the absolute absence or presence - of CD155 at the surface of the cell is critical for its immunomodulatory capabilities ${ }^{50}$. Under physiological conditions, CD155 is expressed at low levels, where a balance between its immune activating and inhibitory functions maintains the normal function of immune cells. Thus, the MAP4K4-dependent increased surface expression of CD155 on growth factor-activated cells could not only affect the migratory potential of the cells but also locally (in the tumor microenvironment) repress immune system activation.

Our findings point towards a role of fast-endophilin mediated endocytosis (FEME) through EndoA 1 in the control of the invasive phenotype of these cells. EndoA1 and A3 are highly expressed in $\mathrm{SHH} M B$ compared to other brain tumors and expression correlates positively with MAP4K4 in this tumor type. This contrasts with Clathrin, which correlates negatively with MAP4K4 in SHH MB and with the lack of clear evidence that clathrin-mediated endocytosis is necessary for migration control in our cell models. The role of endophilins in cancer progression is complex and controversial, as endophilins can both promote and reduce cancer cell migration, depending on tumor type ${ }^{24,59,68}$. We previously found that 5 -( $N$-ethyl- $N$-isopropyl)amiloride (EIPA), a micropinocytosis inhibitor ${ }^{69}$, blocks HGF-induced invasion ${ }^{52}$. However, depletion of RAC1 or CTBP1, two upstream activators of micropinocytosis, did not significantly impact HGF-induced motility. This suggests that the pronounced effect of EIPA could be due to the endocytosis-unrelated effects of this drug on ion transport, intracellular $\mathrm{pH}$ and cytoskeleton regulation, thus affecting FEME indirectly ${ }^{24,59}$. FEME is involved in the endocytosis of activated receptors at the leading edge of migrating cells ${ }^{70}$ and fast and ultrafast endocytosis are important for chemotaxis ${ }^{71}$. FEME could be involved in the internalization of c-MET, thereby controlling signal transmission and persistence of this receptor ${ }^{24}$. Our study identified a possible link between 
MAP4K4 function and FEME through the regulated PM-association of the FEME priming factors SH3KBP1, CIP4 and FBP17. We recently identified a potential interaction of MAP4K4 with SH3KBP1using a Bio-ID based approach ${ }^{60}$, and in this study, we found that MAP4K4 regulates CIP4 PM-association and its accumulation in cortical patches. The early endosome marker EEA1 also localizes to these patches, and it is thus possible that one aspect of FEME processing towards early endosomes occurs preferentially there. Depletion of MAP4K4 prevented EEA1 recruitment to these patches, led to an overall decrease in EEA1-positive vesicles and caused accumulation of CIP4 in this location instead. These phenomena are quite exactly phenocopied by EndoA1 depletion. It is thus possible that MAP4K4 controls FEME by orchestrating a step between FEME priming and trafficking towards early endosomes. Our working model is that MAP4K4 orchestrates PM association of CIP4 from a non-PM-associated subcellular compartment to the "CIP4 patch" in close proximity of or associated with the PM, where also early endosomes enrich. Upon HGF stimulation, CIP4 translocates to the PM, where it is rapidly turned over through EndoA1-dependent FEME before it is recycled back to the PM. Without MAP4K4 function, CIP4 remains associated with the patches, hence overall amount of PM associated CIP4 increases. Some key questions remain: (i) Does the MAP4K4-dependent mechanism of action involve phosphorylation of one of the components involved in FEME, (ii) is MAP4K4 regulation of CIP4 involved in CIP4-dependent recruitment of Endophilin-A1 to the plasma membrane and (iii) does MAP4K4 control of FEME priming involve the activation of CDC42 downstream of GEFs and GAPs. Elucidation of these mechanisms will be essential to explore therapeutic targeting strategies for specifically repressing MAP4K4 controlled pro-migratory endocytosis.

In conclusion we found that the HGF-C-MET-MAP4K4 axis regulates PM association and surface expression of a number of proteins affecting different pro-oncogenic mechanisms, including chemotherapy sensitivity, ion homeostasis and cell migration. Our study furthermore indicates the contribution of endophilin A proteins in invasion control downstream of MAP4K4. It thus highlights kinase regulation of the PM proteomic composition as a novel mechanism involved in the establishment and maintenance of the oncogenic phenotype in MB and other solid tumors. The study provides a conceptual basis for further analysis of the dynamic adaptation of tumor cells to environmental cues by plasma membrane proteome modification. Finally, targeting MAP4K4 controlled endocytic activity could represent a novel druggable vulnerability in medulloblastoma cells to restrict tumor growth and dissemination.

\section{Materials and Methods}

\section{Cell lines and culture conditions}

DAOY human MB cells were purchased from the American Type Culture Collection (ATCC, Rockville, MD, USA). ONS-76 cells were generously provided by Michael Taylor (SickKids, Canada). CNMC-XD760 and MDA-MB-231 cells were generously provided by Javad Nazarian (University Children's Hospital Zurich, Switzerland). DAOY and DAOY-LA-EGFP cells were cultured in iMEM complemented with $10 \%$ FBS. Medium without FBS is used for starvation and further referred to as serum-free medium (SFM). ONS-76-LA-EGFP cells were cultured in RPMI 1640 complemented with 10\% FBS. Medium 
without FBS is used for starvation. DAOY and ONS-76 Lifeact-enhanced green fluorescent protein (LAEGFP) cells were produced by lentiviral transduction of DAOY cells with pLenti-LA-EGFP. Cell line authentication and cross-contamination testing were performed by Multiplexion $\mathrm{GmbH}$ (Heidelberg, Germany) by single nucleotide polymorphism (SNP) profiling.

\section{Animals}

Pregnant Wild type C57BL/6JRj females were purchased from Janvier Labs and were kept in the animal facilities of the University of Zürich Laboratory Animal Centre. Mouse protocols for organotypic brain slice culture were approved by the Veterinary Office of the Canton Zürich.

\section{Generation of CRISPR/Cas9-mediated MAP4K4 knockout cells}

MAP4K4 gene-specific single-guide RNA (sgRNA) were designed using Synthego CRISPR design online tool (https://design.synthego.com). Oligos were synthetized by Microsynth (Balgach, Switzerland) and cloned into LentiCRISPRv2 transfer plasmid (Addgene, 52961) with a single tube restriction and ligation method as described by McComb et al. ${ }^{72}$. Production of lentiviral vectors and cell transduction was performed as described previously ${ }^{14}$. The efficiency of the knockouts was tested by immunoblot. Only the guide with the highest efficiency have been selected for further experiments, sgMAP4K4\#1, exon 7, GGGCGGAGAAATACGTTCAT).

\section{Cell-surface labelling}

DAOY cells were prepared in four T75 flasks to reach a 70\% confluency (per condition and replicates). Standard medium was then replaced with SFM. After 24 hours, cells were $85 \%$ confluent, and media were replaced with either pre-warmed SFM or pre-warmed SFM supplemented with $20 \mathrm{ng} / \mathrm{mL}$ HGF (PeproTech, 100-39) for 30 min. EZ-Link-Sulfo-NHS-SS-biotin kit (Thermo Scientific, 89881) was used to surface label DAOY_sgCTL and DAOY_sgMAP4K4 cells. In brief, $10 \mathrm{ml}$ EZ-Link-Sulfo-NHS-SS (0.25 $\mathrm{mg} / \mathrm{ml}$, diluted in ice-cold PBS) was added at $4^{\circ} \mathrm{C}$ for 30 minutes. Biotinylation reaction was terminated by addition $500 \mu \mathrm{l}$ of provided quenching solution. Cells from four T75 were collected in a unique tube and washed three times with $10 \mathrm{ml}$ ice-cold TBS (700g, 3 minutes centrifugations). Cell pellets were lysed on ice for 30 minutes with one cycle of sonication (Sonoplus, HD2070) and 5 seconds vortexing every 5 minutes. Insoluble debris pelleted using centrifugation at $10^{\prime} 000 \mathrm{~g}$ for 2 minutes. Biotinylated proteins were affinity-purified from lysates using columns containing NeutrAvidin Agarose slurry for one hour at room temperature with end-over-end mixing. After 3 washing steps with lysis buffer, bound proteins were eluted using sample buffer (BioRad, 1610747), complemented with $50 \mathrm{mM}$ DTT, for one hour at room temperature with end-over-end mixing. Purified lysates were kept at $-20^{\circ} \mathrm{C}$ for up to 1 day before Mass spectrometry analysis.

Samples were prepared for mass-spectrometry (MS) by using the iST Kit (PreOmics, Germany) according to a modified protocol. In brief, proteins were precipitated with a $10 \%$ TCA, solubilized in 50 $\mu \mathrm{l}$ iST Kit lysis buffer, boiled at $95^{\circ} \mathrm{C}$ for 10 minutes and processed with High Intensity Focused Ultrasound (HIFU) for $30 \mathrm{~s}$, with ultrasonic amplitude set to $85 \%$. Proteins were quantified using Qubit, and $50 \mu \mathrm{g}$ of the samples were for digestion using $50 \mu \mathrm{l}$ of the iST Kit digestion solution. After $60 \mathrm{~min} \mathrm{f}$ 
incubation at $37^{\circ} \mathrm{C}$, the digestion was stopped with $100 \mu \mathrm{l}$ of iST Kit Stop solution. The non-bound components of the solutions in the cartridge were removed by centrifugation at $3800 \times \mathrm{g}$, while the peptides were retained by the iST-filter. Finally, the peptides were washed, eluted, dried and resolubilized in $20 \mu \mathrm{l}$ iST Kit LC-Load buffer for MS-Analysis.

\section{Liquid chromatography-mass spectrometry analysis}

MS analysis was performed on a Q Exactive HF-X mass spectrometer (Thermo Scientific) equipped with a Digital PicoView source (New Objective) and coupled to a M-Class UPLC (Waters). Solvent composition at the two channels was $0.1 \%$ formic acid for channel $\mathrm{A}$ and $0.1 \%$ formic acid, $99.9 \%$ acetonitrile for channel $B$. For each sample, $1 \mu$ l of peptides were loaded on a commercial MZ Symmetry C18 Trap Column (100A, $5 \mu \mathrm{m}, 180 \mu \mathrm{m} \times 20 \mathrm{~mm}$, Waters) followed by nanoEase MZ C18 HSS T3

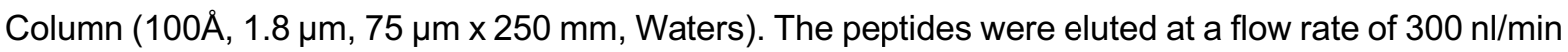
by a gradient from 8 to $27 \%$ B in $85 \mathrm{~min}, 35 \%$ B in $5 \mathrm{~min}$ and $80 \%$ B in $1 \mathrm{~min}$. Samples were acquired in a randomized order. The mass spectrometer was operated in data-dependent mode (DDA), acquiring a full-scan MS spectra (350-1'400 m/z) at a resolution of $120^{\prime} 000$ at $200 \mathrm{~m} / \mathrm{z}$ after accumulation to a target value of 3 '000'000, followed by HCD (higher-energy collision dissociation) fragmentation on the twenty most intense signals per cycle. HCD spectra were acquired at a resolution of 15'000 using a normalized collision energy of 25 and a maximum injection time of $22 \mathrm{~ms}$. The automatic gain control (AGC) was set to 100 '000 ions. Charge state screening was enabled. Singly, unassigned, and charge states higher than seven were rejected. Only precursors with intensity above 110 '000 were selected for MS/MS. Precursor masses previously selected for MS/MS measurement were excluded from further selection for $30 \mathrm{~s}$, and the exclusion window was set at $10 \mathrm{ppm}$. The samples were acquired using internal lock mass calibration on $\mathrm{m} / \mathrm{z} 371.1012$ and 445.1200 . The mass spectrometry proteomics data were handled using the local laboratory information management system (LIMS) ${ }^{73}$.

\section{Protein identification and label free protein quantification}

The acquired raw MS data were processed by MaxQuant (version 1.6.2.3), followed by protein identification using the integrated Andromeda search engine ${ }^{74}$. Spectra were searched against a Swissprot Homo sapiens reference proteome (taxonomy 9606, version from 2016-12-09), concatenated to its reversed decoyed fasta database and common protein contaminants. Carbamidomethylation of cysteine was set as fixed modification, while methionine oxidation and $\mathrm{N}$ terminal protein acetylation were set as variable. Enzyme specificity was set to trypsin/P allowing a minimal peptide length of 7 amino acids and a maximum of two missed cleavages. MaxQuant Orbitrap default search settings were used. The maximum false discovery rate (FDR) was set to 0.01 for peptides and 0.05 for proteins. Label free quantification was enabled and a 2-minute window for match between runs was applied. In the MaxQuant experimental design template, each file is kept separate in the experimental design to obtain individual quantitative values. Protein fold changes were computed based on Intensity values reported in the proteinGroups.txt file. A set of functions implemented in the R package SRMService ${ }^{75}$ was used to filter for proteins with two or more peptides allowing for a maximum of four missing values, and to normalize the data with a modified robust z-score transformation and to 
compute $p$-values using the t-test with pooled variance. If all protein measurements are missing in one of the conditions, a pseudo fold change was computed replacing the missing group average by the mean of $10 \%$ smallest protein intensities in that condition. For data visualization and normalization across all samples, all group comparison correspond has been processed by statistical analysis of all sgMAP4K4 samples (starved and stimulated) versus all sgCTL samples (starved and stimulated).

\section{Chemotherapy sensitivity analysis}

400 DAOY sgCTL or sgMAP4K4 were seeded per well in 384 well microplate and incubated 24 hours at $37^{\circ} \mathrm{C}$. Media were either replaced with low-serum media (1\% FBS) or with fresh standard culture media (10\% FBS) and incubated for another 24 hours at $37^{\circ} \mathrm{C}$. Cells were treated with either low-serum media or low-serum media supplemented with $20 \mathrm{ng} / \mathrm{mL}$ HGF (PeproTech, 100-39) or by standard culture media supplemented with Lomustine (Selleckchem, S1840, $5 \mu \mathrm{M}$ to $100 \mu \mathrm{M}$ ) or Etoposide (Selleckchem, S1225, $1 \mathrm{nM}$ to $10 \mu \mathrm{M}$ ). Cell proliferation was quantified after $48 \mathrm{~h}$ of incubation using cell proliferation reagent WST-1 (Roche, 11644807001) following the manufacturer instructions. Sample absorbance was measured against background using a microplate reader (Biotek Instruments, Cytation 3) and statistically analyzed using Prism software (GraphPad).

\section{Primary tumor gene expression analysis}

Gene expression data were obtained from the R2 genomics and visualization platform (https://r2.amc.nl). Berchtold - 172 - MAS5.0 - u133p2; Normal cerebellum - Roth - 9 - MAS5.0 - u133p2; Tumor Medulloblastoma (SHH) - Pfister - 73 - MAS5.0 - u133p2; Tumor Medulloblastoma - Hsieh - 31 - MAS5.0 - u133p2; Tumor Medulloblastoma - Pfister - 273 - MAS5.0 - u133p2; Tumor Medulloblastoma public - Delattre - 57 - MAS5.0 - u133p2; Tumor Medulloblastoma PLoS One - Kool - 62 - MAS5.0 u133p2; Tumor Medulloblastoma - Gilbertson - 76 - MAS5.0 - u133p2 were used to analyze CLIC1 expression in Medulloblastoma versus normal brain tissues. The Tumor Medulloblastoma - Cavalli 763 - rna_sketch - hugene11t dataset with 763 primary medulloblastoma samples was used for Kaplan Meier survival analysis relative to CLIC1 mRNA expression levels. The Tumor Medulloblastoma Cavalli - 763 - rna_sketch - hugene11t dataset with 763 primary medulloblastoma samples was used. Molecular pathways correlating with MAP4K4 gene expression have been determine using the R2 genomics and visualization platform built-in KEGG PathwayFinder by Gene correlation tool. Transcriptomic and proteomic data were obtained from the ProTrack pediatric brain tumor database of the Clinical proteomic Tumor Analysis Consortium (CPTAC) and Children's Brain Tumor Tissue Consortium (CBTTC).

\section{Patch-clamp analysis}

DAOY sgCTL or sgMAP4K4 were seeded in a $35 \mathrm{~mm}^{2}$ dish to reach $20 \%$ confluency. After 6 hours incubation time in standard culture condition, medium was replaced with starvation medium. The next day, cells were treated with either SFM or SFM supplemented with $20 \mathrm{ng} / \mathrm{ml} \mathrm{HGF}$ for $30 \mathrm{~min}$. Cells were kept in the patch chamber for $30 \mathrm{~min}$. Whole-cell recordings at room temperature were performed on DAOY sgCTL and sgMAP4K4 cells using patch pipettes of $\sim 7 \mathrm{M} \Omega$. The patch pipette solution contained 
(in mM) $95 \mathrm{~K}$-gluconate, $30 \mathrm{KCl}, 4.8 \mathrm{Na}_{2} \mathrm{HPO}_{4}, 1.2 \mathrm{NaH}_{2} \mathrm{PO}_{4}, 5$ glucose, $2.38 \mathrm{MgCl}_{2}, 0.76 \mathrm{CaCl}_{2}, 1$ EGTA, 3 K-ATP. The bath solution contained (in $\mathrm{mM}$ ) $142 \mathrm{NaCl}, 1.8 \mathrm{MgCl}_{2}, 1.8 \mathrm{CaCl}_{2}, 10 \mathrm{HEPES}, 3$ $\mathrm{KCl}$. Compensations and measurements were done within 30 seconds after obtaining whole-cell to avoid drastic changes in cytoplasmic ionic concentration.

\section{Gene annotation and pathway enrichment analysis}

Gene annotation and pathway enrichment analysis on the surface-proteomics results were performed using the Metascape webtool (https://metascape.org) developed by Zhou et al. ${ }^{46}$. Transmembrane proteins were annotated using TMHMM prediction algorithm ${ }^{29}$. We performed pathway enrichment using all pathway enrichment categories, standard settings and using all proteins identified by massspectrometry as background genes.

\section{siRNA transfections}

100 '000 DAOY or ONS-76 cells were seeded per well in 6-well plates. The following day, cells were transfected with $10 \mathrm{nM}$ siRNAs using Lipofectamine RNAiMAX Transfection Reagent (Invitrogen, 13778075) plus. siRNA used are referenced in Table S1. After 6 hours, the media were changed, and cells were kept in culture overnight for downstream analyses.

\section{qRT-PCR analysis of gene expression}

100 '000 siRNA transfected DAOY cells ( $24 \mathrm{~h}$ after transfection) were seeded per well in 6 well plates and incubated overnight. For quantitative real-time PCR (qRT-PCR) analysis of target genes, total RNA was isolated using Qiagen RNeasy Mini Kit (Qiagen, 74106). $1 \mu \mathrm{g}$ of mRNA was converted to cDNA using the high-capacity cDNA Reverse Transcription Kit (Applied Biosystems, 4368813). qRT-PCR was performed using PowerUp Syber Green (Applied Biosystems, A25776) under conditions optimized for the ABI7900HT instrument. Primers used are referenced in table S2. The $\Delta \Delta C T$ method was used to calculate the relative gene expression of each gene of interest.

\section{Flow cytometry analysis of CD155 surface expression}

300 '000 siRNA transfected DAOY cells were seeded 24h after transfection per well in low adhesion 6 well plate (Greiner Bio-one, 657970) and incubated for $6 \mathrm{~h}$ in SFM. Cells were then treated with either pre-warmed SFM, or SFM supplemented with $20 \mathrm{ng} / \mathrm{ml} \mathrm{HGF}$ for $30 \mathrm{~min}$. After stimulation, cells were fixed by adding paraformaldehyde pre-warmed to $37^{\circ} \mathrm{C}$ to a final concentration of $4 \%$ and incubated for 20 min at $37^{\circ} \mathrm{C}$. After one wash with PBS supplemented with $2 \% \mathrm{FBS}$, cells were stained with PElabelled anti-human CD155 (BioLegend, 337610, 1:300), corresponding isotype control antibody (BioLegend, 400114, 1:300), or left unstained for $20 \mathrm{~min}$ on ice. Samples were washed with PBS supplemented with $2 \%$ FBS, resuspended in PBS and acquired using BD LSRFortessa flow cytometer (BD Bioscience). Fluorescence levels were analyzed using FlowJo software (BD Bioscience).

\section{Spheroid invasion assay}


2'500 siRNA transfected cells were seeded $24 \mathrm{~h}$ after transfection per well in $100 \mu \mathrm{l}$ of standard growth media in cell-repellent 96 well microplates (Greiner Bio-one, 650790). Plates were incubated 24 hours at $37^{\circ} \mathrm{C}$ in $5 \% \mathrm{CO}_{2}$ to allow spheroid formation. $70 \mu \mathrm{l}$ of culture media were removed from each well and the remaining medium containing the spheroids was complemented with a collagen solution $(2.5 \%$ Pure Col Collagen I, DMEM 1x (Sigma, D2429) and 0.4\% Sodium bicarbonate (Sigma, S8761)). After collagen I polymerization ( 2 hours), fresh $100 \mu \mathrm{l} \mathrm{SFM} \mathrm{+/-} \mathrm{HGF} 20 \mathrm{ng} / \mathrm{ml}$ for DAOY spheroids or standard culture media for ONS-76 spheroids was added per well. Cells were incubated at $37^{\circ} \mathrm{C}$ for 16 hours until fixation with 4\% paraformaldehyde. A 1:5000 dilution of Hoechst (Sigma Aldrich, B2883) was added to stain DNA for nuclei visualization. Images of spheroids and invaded cells were acquired on an Operetta CLS High-Content Analysis System (PerkinElmer, HH16000000) at 5x magnification using the Dapi $(405 \mathrm{~nm})$ channel. Spheroids were localized in the well by acquiring $3 \mathrm{z}$-planes with 250 $\mu \mathrm{m}$ z-spacing. Localized spheroids were re-scanned by acquiring $16 \mathrm{Hoechst-stained} \mathrm{z-planes} \mathrm{with} 25$ $\mu \mathrm{m} z$-spacing and a maximum intensity projection of each spheroid was computed and used for further analysis. Using Harmony software (PerkinElmer), spheroids and invading cells were delineated based on fluorescence threshold. The distance from the center of the spheroid was calculated, and the sum of the cell invasion was determined as the sum of all individual values acquired per well.

\section{Single cell motility assay}

1'000 siRNA transfected ONS-76-LA-EGFP cells were seeded in 96 well microplate (Greiner Bio-one, 655098) previously coated with Pure Col Collagen I (Advanced BioMatrix, 5005) at $10 \mu \mathrm{g} \cdot \mathrm{cm}^{-2}$. After 24 $\mathrm{h}$ incubation under standard culture conditions, cell motility was acquired using temperature $\left(37^{\circ} \mathrm{C}\right)$ and $\mathrm{CO}_{2}(5 \%)$ controlled Operetta CLS High-Content Analysis System (PerkinElmer, HH16000000) (nonconfocal, 20x objective, 4 fields per well, $3 \mathrm{~min}$ intervals, $3 \mathrm{~h}$ acquisition). Cell segmentation was performed using the LA-EGFP channel. Cell speed, area, circularity, and the width/length ratio were calculated over time using Harmony software (PerkinElmer).

\section{Ex vivo Organotypic Cerebellum Slice Culture (OCSC)}

Ex vivo Organotypic Cerebellum Slice Culture was carried out essentially as described previously ${ }^{56}$. Wild type C57BL/6JRj mice were sacrificed at postnatal day 8-10. Cerebella were dissected and kept in ice-cold Geys balanced salt solution containing kynurenic acid (GBSSK) and then embedded in $2 \%$ low melting point agarose gel. $350 \mu \mathrm{m}$ thick sections were cut using a vibratome (Leica, VT1200S) and transferred on inserts (Merck Millipore, PICM03050) for further in vitro culture. Slices were kept in culture and monitored for 15 days, and media were changed daily for the first week and once in two days thereafter. Spheroids of DAOY-LA-EGFP or ONS-76-LA-EGFP cells transfected with siCTL or CD155 were implanted and then grown in the slices for 48 hours. For HGF stimulation, the feeding medium was supplemented with $20 \mathrm{ng} / \mathrm{mL}$ HGF. After treatment, the co-cultured slices were fixed with $4 \%$ paraformaldehyde and washed three times with PBS. Inserts were incubated in standard cell culture trypsin EDTA and incubated at $37^{\circ} \mathrm{C}$ in a humidified incubator for $23 \mathrm{~min}$. After 3 washes, the slices were blocked in PBS containing $3 \%$ fetal calf serum, $3 \%$ bovine serum albumin (BSA), and $0.3 \%$ Triton $\times 100$ for 1 hour at room temperature. Primary anti-Calbindin (Abcam, ab108404, 1:1000) and 
anti-human nuclei (Merck, MAB4383, 1:250) were diluted in the blocking solution and incubated overnight on a shaker at $4^{\circ} \mathrm{C}$. Proliferative cells were detected using the Click-iT EdU cell proliferation kit following (Invitrogen, C10340). Unbound primary antibody was removed with 3 washes with PBS supplemented with 3\% BSA at RT. Secondary antibodies (Table S3) were incubated for $3 \mathrm{~h}$ at RT. The inserts were flat mounted in glycergel mounting medium (Dako, C0563). Image acquisition was performed on a SP8 Leica confocal microscope (Leica Microsystems) and analyzed using Imaris (Oxford Instruments) and ImageJ (Fiji) software.

\section{Immunoblotting (IB)}

Immunoblot was carried out as described $\mathrm{in}^{14}$. DAOY cells were prepared in 6 well plates to reach $70 \%$ confluency within $24 \mathrm{~h}$. Standard media were replaced with SFM. After $24 \mathrm{~h}$ at $85 \%$ confluency, cells were treated with either SFM or SFM supplemented with $20 \mathrm{ng} / \mathrm{ml} \mathrm{HGF}$ (PeproTech, 100-39) for 30 min. Cells lysis was performed with RIPA buffer for $15 \mathrm{~min}$ and ice and cleared lysates were analyzed by SDS-PAGE. Protein concentration was assessed using the Pierce BCA Protein Assay Kit (Thermo Fisher Scientific) according to the manufacturer's instructions. Proteins were separated on Mini-Protean TGX (4-20\%) SDS-PAGE gel (Bio-Rad) and transferred to PVDF membranes (Bio-Rad). Following $1 \mathrm{~h}$ of blocking with $5 \%$ non-fat milk, membranes were probed with primary antibodies listed in Supplementary material (Table S3). GAPDH was used as internal loading control. HRP-linked secondary antibodies (1:2000) were used to detect the primary antibodies. Chemiluminescence detection was carried out using ChemiDoc Touch Gel imaging system (Bio-Rad). The integrated density of detected bands was quantified using Image Lab software (Bio-Rad).

\section{Boyden chamber assay}

Transwell with $5 \mu \mathrm{m}$ pore size (Sarstedt, 83.3932.500) were coated with $0.07 \mu \mathrm{g} / \mu \mathrm{l}$ Pure Col Collagen I (Advanced BioMatrix, 5005) dissolved in $70 \% \mathrm{EtOH}$ for a final concentration of $10 \mu \mathrm{g} . \mathrm{cm}^{-2} .7^{\prime} 500$ transfected cells ( $24 \mathrm{~h}$ after transfection) were resuspended in serum-free medium and seeded in the upper chamber of a 24 well plate containing the coated transwell. Medium in the lower chamber was either serum-free medium or serum-free medium supplemented with $20 \mathrm{ng} / \mathrm{ml} \mathrm{HGF}$ (PeproTech, 10039). Transwell migration was allowed for 18 hours, and cells were then fixed in $4 \%$ paraformaldehyde (Thermo Scientific, 28908) in PBS for 10 min. Fixed cells were stained with DAPI for 15 min. Remaining non-invading cells on the upper surface of the membrane were removed using a cotton swab. Images of DAPI-stained nuclei were acquired using an Axio Observer 2 plus fluorescence microscope (Zeiss) at $5 x$ magnification. Nuclei were counted on the membrane areas using ImageJ software and plotted on Prism 9 software (GraphPad).

\section{Cell proliferation assay}

1'000 DAOY cells were seeded in 96 well plates (Greiner Bio-One, 655087). The following day, cells were transfected according to the protocol described above. Six hours after transfection, medium was replaced with serum-free medium. For timepoint 0 , WST reagent was added to the corresponding well and incubated $30 \mathrm{~min}$ at $37^{\circ} \mathrm{C}$. Absorbance at $440 \mathrm{~nm}$ was measured using a microplate reader (Biotek 
Instruments, Cytation 3). $20 \mathrm{ng} / \mathrm{ml} \mathrm{HGF}$ was added to remaining timepoints and incubated for 24,48 , and 72 hours. At the end of each timepoint, WST reagent was added to corresponding well and absorbance measured. Each day media was replaced with fresh serum-free medium supplemented with HGF. Changes in absorbance over time were analyzed using Prism 9 software (GraphPad).

\section{Immunofluorescence analysis}

1'500 transfected cells ( $24 \mathrm{~h}$ after transfection) were seeded in 384 well plate (Greiner Bio-One, 781090) and incubated overnight in normal growth conditions. Cells were starved in low serum condition $(1 \%$ FBS) for 24 hours and treated with $20 \mathrm{ng} / \mathrm{ml} \mathrm{HGF}$ for 10 - or $30-\mathrm{min}$. Cells were fixed directly with $4 \%$ PFA at $37^{\circ} \mathrm{C}$ for $20 \mathrm{~min}$. Between all subsequent steps, cells were washed five times with PBS using the 50 TS washer (BioTek). Cells were permeabilized with $0.05 \%$ saponin for 20 min at RT. Blocking was performed during 1 hour at RT with 1\% FBS. Primary antibody (Table S3) solution was added to sample and shaken at $100 \mathrm{rpm}$ for 2 hours. Secondary antibody (Table S3) solutions were added to sample and shaken at $100 \mathrm{rpm}$ for 2 hours. Nucleic acids were stained with Hoechst-33342 (Sigma Aldrich, B2883, 1:2000 in PBS) for 20 min at RT. Image acquisition was performed using the Operetta CLS High-Content Analysis System (PerkinElmer, HH16000000) at 40x magnification. 9 fields per well with $9 \mathrm{z}$-planes with $500 \mathrm{~nm}$ z-spacing were acquired per site. Maximum intensity projection was computed and used for subsequent analysis. For puncta quantification, EEA1 or LAMP1 signals were segmented using the Harmony software (PerkinElmer) and quantified. Total number of puncta was referred to the number of cells present per field.

\section{Statistical analysis}

The samples sizes and statistical tests were selected based on previous studies with similar methodologies. Sample sizes were not determined using statistical methods. Statistical analysis was performed using the Prism 9 software (GraphPad). One-way ANOVA repeated measures test using Bonferroni's multiple comparison was performed for multiple comparisons. P-Value $<0.05$ were considered significant $\left({ }^{*}=p<0.05,{ }^{* *}=p<0.01,{ }^{* * *}=p<0.001,{ }^{* * * *}=p<0.0001\right.$, ns $=$ not significant $)$.

\section{Author Contributions}

C.C. and M.B. designed the study, prepared the figures, and wrote the manuscript. C.C. planned all the experiments. C.C. conducted all the experiments unless mentioned otherwise. J.M. generated the CRISPR-Cas9 MAP4K4 cells. L.R. performed the Boyden chamber siRNA screening. M.Z. contributed to the immunofluorescence and IB sample preparation. D.P. performed the patch clamping experiments and S.N. contributed to the planning and evaluation of patch clamping experiments. J.M. and A.G. generated samples and provided experimental support for ex vivo experiments. M.G. helped drafting the study. M.G. and M.B. managed funding acquisition.

\section{Acknowledgments}

We thank Dr. Paolo Nanni, Dr. Jonas Grossman and Claudia Fortes from the Functional Genomic Center Zürich for their help and support for Mass Spectrometry analysis. We like to thank the UZH 
electrophysiology facility (e-phac) for their technical support. Imaging was performed with equipment maintained by the Center for Microscopy and Image Analysis, University of Zurich. This study was supported by grants from the Swiss National Science Foundation (SNF_31003A_165860/1, SNF_310030_188793) to MB and from the Childhood Cancer Foundation to MG.

\section{References}

1. Almén, M. S., Nordström, K. J. V, Fredriksson, R. \& Schiöth, H. B. Mapping the human membrane proteome: a majority of the human membrane proteins can be classified according to function and evolutionary origin. BMC Biol. 7, 50 (2009).

2. Ye, X., Kaczmarczyk, J. A., Luke, B., Saul, R. G., Whiteley, G. R., Nissley, D. V \& Blonder, J. Cell surface protein enrichment for biomarker and drug target discovery using mass spectrometry-based proteomics. in Proteomic and Metabolomic Approaches to Biomarker Discovery 409-420 (Elsevier, 2020). doi:10.1016/B978-0-12-818607-7.00024-4.

3. Orlando, K. \& Guo, W. Membrane Organization and Dynamics in Cell Polarity. Cold Spring Harb. Perspect. Biol. 1, a001321-a001321 (2009).

4. Rodriguez-Furlan, C., Minina, E. A. \& Hicks, G. R. Remove, Recycle, Degrade: Regulating Plasma Membrane Protein Accumulation. Plant Cell 31, 2833-2854 (2019).

5. Schmid, S. L. Reciprocal regulation of signaling and endocytosis: Implications for the evolving cancer cell. J. Cell Biol. 216, 2623-2632 (2017).

6. Hanahan, D. \& Weinberg, R. A. Hallmarks of Cancer: The Next Generation. Cell 144, 646-674 (2011).

7. Mosesson, Y., Mills, G. B. \& Yarden, Y. Derailed endocytosis: an emerging feature of cancer. Nat. Rev. Cancer 8, 835-850 (2008).

8. Skowron, P., Ramaswamy, V. \& Taylor, M. D. Genetic and molecular alterations across medulloblastoma subgroups. J. Mol. Med. 93, 1075-1084 (2015).

9. Northcott, P. A., Korshunov, A., Witt, H., Hielscher, T., Eberhart, C. G., Mack, S., Bouffet, E., Clifford, S. C., Hawkins, C. E., French, P., Rutka, J. T., Pfister, S. \& Taylor, M. D. Medulloblastoma comprises four distinct molecular variants. J. Clin. Oncol. 29, 1408-1414 (2011).

10. Northcott, P. A., Dubuc, A. M., Pfister, S. \& Taylor, M. D. Molecular subgroups of medulloblastoma. Expert Rev. Neurother. 12, 871-884 (2012).

11. Hovestadt, V., Ayrault, O., Swartling, F. J., Robinson, G. W., Pfister, S. M. \& Northcott, P. A. Medulloblastomics revisited: biological and clinical insights from thousands of patients. Nat. Rev. Cancer 20, 42-56 (2020).

12. Aldape, K. et al. Challenges to curing primary brain tumours. Nat. Rev. Clin. Oncol. 16, 509520 (2019).

13. Santhana Kumar, K., Tripolitsioti, D., Ma, M., Grählert, J., Egli, K. B., Fiaschetti, G., Shalaby, T., Grotzer, M. A. \& Baumgartner, M. The Ser/Thr kinase MAP4K4 drives c-Met-induced motility and invasiveness in a cell-based model of SHH medulloblastoma. Springerplus 4, (2015). 
14. MAP4K4 controlled integrin beta1 activation and c-Met endocytosis are associated with invasive behavior of medulloblastoma cells. Oncotarget (2018).

15. Hood, J. D. \& Cheresh, D. A. Role of integrins in cell invasion and migration. Nat. Rev. Cancer 2, 91-100 (2002).

16. de Franceschi, N., Hamidi, H., Alanko, J., Sahgal, P. \& Ivaska, J. Integrin traffic-the update. J. Cell Sci. 128, 839-852 (2015).

17. Kermorgant, S., Zicha, D. \& Parker, P. J. PKC controls HGF-dependent c-Met traffic, signalling and cell migration. EMBO J. 23, 3721-3734 (2004).

18. Alanko, J., Mai, A., Jacquemet, G., Schauer, K., Kaukonen, R., Saari, M., Goud, B. \& Ivaska, J. Integrin endosomal signalling suppresses anoikis. Nat. Cell Biol. 17, 1412-1421 (2015).

19. Kjaerulff, O., Brodin, L. \& Jung, A. The Structure and Function of Endophilin Proteins. Cell Biochem. Biophys. 60, 137-154 (2011).

20. Masuda, M., Takeda, S., Sone, M., Ohki, T., Mori, H., Kamioka, Y. \& Mochizuki, N. Endophilin BAR domain drives membrane curvature by two newly identified structure-based mechanisms. EMBO J. 25, 2889-2897 (2006).

21. Ferreira, A. P. A. \& Boucrot, E. Mechanisms of Carrier Formation during Clathrin-Independent Endocytosis. Trends Cell Biol. 28, 188-200 (2018).

22. Verstreken, P., Kjaerulff, O., Lloyd, T. E., Atkinson, R., Zhou, Y., Meinertzhagen, I. A. \& Bellen, H. J. Endophilin Mutations Block Clathrin-Mediated Endocytosis but Not Neurotransmitter Release. Cell 109, 101-112 (2002).

23. Gad, H., Ringstad, N., Löw, P., Kjaerulff, O., Gustafsson, J., Wenk, M., Di Paolo, G., Nemoto, Y., Crum, J., Ellisman, M. H., De Camilli, P., Shupliakov, O. \& Brodin, L. Fission and Uncoating of Synaptic Clathrin-Coated Vesicles Are Perturbed by Disruption of Interactions with the $\mathrm{SH} 3$ Domain of Endophilin. Neuron 27, 301-312 (2000).

24. Boucrot, E., Ferreira, A. P. A., Almeida-Souza, L., Debard, S., Vallis, Y., Howard, G., Bertot, L., Sauvonnet, N. \& McMahon, H. T. Endophilin marks and controls a clathrin-independent endocytic pathway. Nature 517, 460-465 (2015).

25. Genet, G., Boyé, K., Mathivet, T., Ola, R., Zhang, F., Dubrac, A., Li, J., Genet, N., Henrique Geraldo, L., Benedetti, L., Künzel, S., Pibouin-Fragner, L., Thomas, J.-L. \& Eichmann, A. Endophilin-A2 dependent VEGFR2 endocytosis promotes sprouting angiogenesis. Nat. Commun. 10, 2350 (2019).

26. Poudel, K. R., Roh-Johnson, M., Su, A., Ho, T., Mathsyaraja, H., Anderson, S., Grady, W. M., Moens, C. B., Conacci-Sorrell, M., Eisenman, R. N. \& Bai, J. Competition between TIAM1 and Membranes Balances Endophilin A3 Activity in Cancer Metastasis. Dev. Cell 45, 738--752.e6 (2018).

27. Giachino, C., Lantelme, E., Lanzetti, L., Saccone, S., Valle, G. Della \& Migone, N. A Novel SH3Containing Human Gene Family Preferentially Expressed in the Central Nervous System. Genomics 41, 427-434 (1997).

28. Itzhak, D. N., Davies, C., Tyanova, S., Mishra, A., Williamson, J., Antrobus, R., Cox, J., Weekes, M. P. \& Borner, G. H. H. A Mass Spectrometry-Based Approach for Mapping Protein Subcellular 
Localization Reveals the Spatial Proteome of Mouse Primary Neurons. Cell Rep. 20, 2706-2718 (2017).

29. Moller, S., Croning, M. D. R. \& Apweiler, R. Evaluation of methods for the prediction of membrane spanning regions. Bioinformatics 17, 646-653 (2001).

30. Itzhak, D. N., Tyanova, S., Cox, J. \& Borner, G. H. Global, quantitative and dynamic mapping of protein subcellular localization. Elife 5, 1-36 (2016).

31. Huang, Y., Anderle, P., Bussey, K. J., Barbacioru, C., Shankavaram, U., Dai, Z., Reinhold, W. C., Papp, A., Weinstein, J. N. \& Sadée, W. Membrane transporters and channels: Role of the transportome in cancer chemosensitivity and chemoresistance. Cancer Res. 64, 4294-4301 (2004).

32. Rubino, S., Bach, M. D., Schober, A. L., Lambert, I. H. \& Mongin, A. A. Downregulation of leucine-rich repeat-containing $8 \mathrm{~A}$ limits proliferation and increases sensitivity of glioblastoma to temozolomide and carmustine. Front. Oncol. 8, 1-12 (2018).

33. Gao, J., Zheng, Q., Xin, N., Wang, W. \& Zhao, C. CD155, an onco-immunologic molecule in human tumors. Cancer Sci. 108, 1934-1938 (2017).

34. Picarda, E., Ohaegbulam, K. C. \& Zang, X. Molecular pathways: Targeting B7-H3 (CD276) for human cancer immunotherapy. Clin. Cancer Res. 22, 3425-3431 (2016).

35. Curigliano, G., Criscitiello, C., Gelao, L. \& Goldhirsch, A. Molecular pathways: Human leukocyte antigen G(HLA-G). Clin. Cancer Res. 19, 5564-5571 (2013).

36. Morishita, K., Watanabe, K. \& Ichijo, H. Cell volume regulation in cancer cell migration driven by osmotic water flow. Cancer Sci. 110, 2337-2347 (2019).

37. Francisco, M. A. et al. Chloride intracellular channel 1 cooperates with potassium channel EAG2 to promote medulloblastoma growth. J. Exp. Med. 217, 324-387 (2020).

38. Liu, F., Zhang, T., Zou, S., Jiang, B. \& Hua, D. B7-H3 promotes cell migration and invasion through the Jak2/Stat3/MMP9 signaling pathway in colorectal cancer. Mol. Med. Rep. 12, 54555460 (2015).

39. Hoffmann, E. K. \& Lambert, I. H. Ion channels and transporters in the development of drug resistance in cancer cells. Philos. Trans. R. Soc. B Biol. Sci. 369, 20130109 (2014).

40. Giacomini, K. M. et al. Membrane transporters in drug development. Nat. Rev. Drug Discov. 9, 215-236 (2010).

41. Lagas, J. S., Fan, L., Wagenaar, E., Vlaming, M. L. H., van Tellingen, O., Beijnen, J. H. \& Schinkel, A. H. P-glycoprotein (P-gp/Abcb1), Abcc2, and Abcc3 Determine the Pharmacokinetics of Etoposide. Clin. Cancer Res. 16, 130-140 (2010).

42. Deeley, R. G., Westlake, C. \& Cole, S. P. C. Transmembrane Transport of Endo- and Xenobiotics by Mammalian ATP-Binding Cassette Multidrug Resistance Proteins. Physiol. Rev. 86, 849-899 (2006).

43. Sørensen, B. H., Nielsen, D., Thorsteinsdottir, U. A., Hoffmann, E. K. \& Lambert, I. H. Downregulation of LRRC8A protects human ovarian and alveolar carcinoma cells against Cisplatin-induced expression of p53, MDM2, p21 Waf1/Cip1, and Caspase-9/-3 activation. Am. J. Physiol. Physiol. 310, C857-C873 (2016). 
44. Packer, R. J. et al. Outcome for children with medulloblastoma treated with radiation and cisplatin, CCNU, and vincristine chemotherapy. J. Neurosurg. 81, 690-698 (1994).

45. Greenberg, H. S., Chamberlain, M. C., Glantz, M. J. \& Wang, S. Adult medulloblastoma: Multiagent chemotherapy. Neuro. Oncol. 3, 29-34 (2001).

46. Zhou, Y., Zhou, B., Pache, L., Chang, M., Khodabakhshi, A. H., Tanaseichuk, O., Benner, C. \& Chanda, S. K. Metascape provides a biologist-oriented resource for the analysis of systemslevel datasets. Nat. Commun. 10, (2019).

47. Thul, P. J. et al. A subcellular map of the human proteome. Science (80-. ). 356 , eaal3321 (2017).

48. DEFELICE, L. Transporter structure and mechanism. Trends Neurosci. 27, 352-359 (2004).

49. Payne, S. L., Levin, M. \& Oudin, M. J. Bioelectric Control of Metastasis in Solid Tumors. Bioelectricity 1, 114-130 (2019).

50. Kučan Brlić, P., Lenac Roviš, T., Cinamon, G., Tsukerman, P., Mandelboim, O. \& Jonjić, S. Targeting PVR (CD155) and its receptors in anti-tumor therapy. Cell. Mol. Immunol. 16, 40-52 (2019).

51. Neve, A., Kumar, K. S., Tripolitsioti, D., Grotzer, M. A. \& Baumgartner, M. Investigation of brain tissue infiltration by medulloblastoma cells in an ex vivo model. Sci. Rep. 7, (2017).

52. Tripolitsioti, D., Kumar, K. S., Neve, A., Migliavacca, J., Capdeville, C., Rushing, E. J., Ma, M., Kijima, N., Sharma, A., Pruschy, M., McComb, S., Taylor, M. D., Grotzer, M. A. \& Baumgartner, M. MAP4K4 controlled integrin $\beta 1$ activation and c-Met endocytosis are associated with invasive behavior of medulloblastoma cells. Oncotarget 9, (2018).

53. Cavalli, F. M. G. et al. Intertumoral Heterogeneity within Medulloblastoma Subgroups. Cancer Cell 31, 737--754.e6 (2017).

54. Petralia, F. et al. Integrated Proteogenomic Characterization across Major Histological Types of Pediatric Brain Cancer. Cell 183, 1962-1985.e31 (2020).

55. Cendrowski, J., Mamińska, A. \& Miaczynska, M. Endocytic regulation of cytokine receptor signaling. Cytokine Growth Factor Rev. 32, 63-73 (2016).

56. Liberali, P., Kakkonen, E., Turacchio, G., Valente, C., Spaar, A., Perinetti, G., Böckmann, R. A., Corda, D., Colanzi, A., Marjomaki, V. \& Luini, A. The closure of Pak1-dependent macropinosomes requires the phosphorylation of CtBP1/BARS. EMBO J. 27, 970-981 (2008).

57. Kumar, K. S., Pillong, M., Kunze, J., Burghardt, I., Weller, M., Grotzer, M. A., Schneider, G. \& Baumgartner, M. Computer-assisted quantification of motile and invasive capabilities of cancer cells. Sci. Rep. 5, 15338 (2015).

58. Roper, S. J., Linke, F., Scotting, P. J. \& Coyle, B. 3D spheroid models of paediatric SHH medulloblastoma mimic tumour biology, drug response and metastatic dissemination. Sci. Rep. 1-17 (2021) doi:10.1038/s41598-021-83809-6.

59. Casamento, A. \& Boucrot, E. Molecular mechanism of Fast Endophilin-Mediated Endocytosis. Biochem. J. 477, 2327-2345 (2020).

60. Migliavacca, J., Züllig, B., Capdeville, C., Grotzer, M. \& Baumgartner, M. The MAP4K4STRIPAK complex promotes growth and tissue invasion in medulloblastoma. bioRxiv 
2021.05.07.442906 (2021) doi:10.1101/2021.05.07.442906.

61. Tian, L., Nelson, D. L. \& Stewart, D. M. Cdc42-interacting Protein 4 Mediates Binding of the Wiskott-Aldrich Syndrome Protein to Microtubules. J. Biol. Chem. 275, 7854-7861 (2000).

62. Dozmorov, M. G., Hurst, R. E., Culkin, D. J., Kropp, B. P., Frank, M. B., Osban, J., Penning, T. M. \& Lin, H.-K. Unique patterns of molecular profiling between human prostate cancer LNCaP and PC-3 cells. Prostate 69, 1077-1090 (2009).

63. Pollak, J., Rai, K. G., Funk, C. C., Arora, S., Lee, E., Zhu, J., Price, N. D., Paddison, P. J., Ramirez, J.-M. \& Rostomily, R. C. Ion channel expression patterns in glioblastoma stem cells with functional and therapeutic implications for malignancy. PLoS One 12, e0172884 (2017).

64. Sloan, K. E., Stewart, J. K., Treloar, A. F., Matthews, R. T. \& Jay, D. G. CD155/PVR enhances glioma cell dispersal by regulating adhesion signaling and focal adhesion dynamics. Cancer Res. 65, 10930-10937 (2005).

65. Zheng, Q., Gao, J., Yin, P., Wang, W., Wang, B., Li, Y. \& Zhao, C. CD155 contributes to the mesenchymal phenotype of triple-negative breast cancer. Cancer Sci. 111, 383-394 (2020).

66. Okumura, G., Iguchi-Manaka, A., Murata, R., Yamashita-Kanemaru, Y., Shibuya, A. \& Shibuya, K. Tumor-derived soluble CD155 inhibits DNAM-1-mediated antitumor activity of natural killer cells. J. Exp. Med. 217, (2020).

67. He, W., Zhang, H., Han, F., Chen, X., Lin, R., Wang, W., Qiu, H., Zhuang, Z., Liao, Q., Zhang, W., Cai, Q., Cui, Y., Jiang, W., Wang, H. \& Ke, Z. CD155T/TIGIT Signaling Regulates CD8+ Tcell Metabolism and Promotes Tumor Progression in Human Gastric Cancer. Cancer Res. 77, 6375-6388 (2017).

68. Behavior, M., Majumdar, S., Gong, E. M., Vizio, D. Di, Dreyfuss, J., Degraff, D. J., Hager, M. H., Park, P. J., Bellmunt, J., Matusik, R. J., Rosenberg, J. E. \& Adam, R. M. Loss of Sh3gl2 I Endophilin A1 Is a Common Event in Urothelial Carcinoma that Promotes. 15, 749-760 (2013).

69. Kabayama, H., Takeuchi, M., Taniguchi, M., Tokushige, N., Kozaki, S., Mizutani, A., Nakamura, T. \& Mikoshiba, K. Syntaxin 1B suppresses macropinocytosis and semaphorin 3A-induced growth cone collapse. J. Neurosci. 31, 7357-7364 (2011).

70. Mondal, S., Powers, I., Narayan, K., Botterbusch, S. \& Baumgart, T. Endophilin Recruitment via GPCR Interactions Enables Membrane Curvature Generation in the Absence of Anionic Lipids. bioRxiv 2020.07.11.198937 (2020) doi:10.1101/2020.07.11.198937.

71. Watanabe, S. \& Boucrot, E. Fast and ultrafast endocytosis. Curr. Opin. Cell Biol. 47, 64-71 (2017).

72. McComb, S., Aguadé-Gorgorió, J., Harder, L., Marovca, B., Cario, G., Eckert, C., Schrappe, M., Stanulla, M., Von Stackelberg, A., Bourquin, J. P. \& Bornhauser, B. C. Activation of concurrent apoptosis and necroptosis by SMAC mimetics for the treatment of refractory and relapsed ALL. Sci. Transl. Med. 8, (2016).

73. Türker, C., Akal, F., Joho, D., Panse, C., Barkow-Oesterreicher, S., Rehrauer, H. \& Schlapbach, R. B-Fabric. in Proceedings of the 13th International Conference on Extending Database Technology - EDBT '10 717 (ACM Press, 2010). doi:10.1145/1739041.1739135.

74. Cox, J. \& Mann, M. MaxQuant enables high peptide identification rates, individualized p.p.b.- 
range mass accuracies and proteome-wide protein quantification. Nat. Biotechnol. 26, 13671372 (2008).

75. Wolski, W., Grossmann, J. \& Panse, C. SRMService - R-Package to Report Quantitative Mass Spectrometry Data. (2018).

76. Falsig, J. \& Aguzzi, A. The prion organotypic slice culture assay-POSCA. Nat. Protoc. 3, 555562 (2008).

\section{Figure legends}

\section{Figure 1}

HGF-C-MET signaling alters composition of plasma membrane (PM)-associated proteome. (A) General workflow for cell-surface protein isolation, mass spectrometry and computational analysis used. (B) log2 Fold change of TM proteins comparisons between treatments. Green: positive fold change (increased PM-association), red: negative fold change (decreased PM-association. (C) Volcano plot of PM-associated proteins identified by deep mass-spectrometry analysis. Red colored dots indicate proteins with predicted PM localization. sgCTL: CRISPR/CAS-9 control guide RNA expressing cells; sgMAP4K4: CRISPR/CAS-9 MAP4K4-specific guide RNA expressing cells; + HGF: SFM supplemented with $20 \mathrm{ng} / \mathrm{ml} \mathrm{HGF}$ for $30 \mathrm{~min}$. (D) Quantification of the PM association of different receptor tyrosine kinases. Box plots display normalized z-score values extracted from all-group comparison of MS analysis. $N=4$ independent experiments, mean \& SD, ${ }^{*}=p<0.05$. (E) Heatmaps of enriched ontology clusters across multiple two-group analyses of significantly altered, PM-annotated proteins with positive (upper) or negative (lower) fold change. Panel colors are defined by p-values. Gray cells: No enrichment detected for that term in the corresponding two-group analyses. (F) Overview of a selection of potentially clinically relevant PM-associated proteins. Yellow bar: no change; red arrow: decreased PM association; green arrow: increased PM association

\section{Figure 2}

MAP4K4 modulates the ion homeostasis of DAOY cells. (A) Quantification of PM association of proteins involved in ion homeostasis. Box plots display normalized z-score values extracted from allgroup comparison of MS analysis. $\mathrm{N}=4$ independent experiments, mean \& SD, ${ }^{*}=p<0.05,{ }^{* *}=p<$ 0.01 . (B) Pathway enrichment analysis of significantly altered transmembrane-proteins derived from two-group analysis comparing DAOY sgCTL and sgMAP4K4 +/- HGF (20 ng/ml, $30 \mathrm{~min})$. (C) Quantification of proteins associated with GO Potassium ion homeostasis. Box plots display normalized z-score values extracted from all-group comparison of MS analysis. $N=4$ independent experiment, mean \& SD, ${ }^{*}=p<0.05,{ }^{* *}=p<0.01,{ }^{* * *}=p<0.001,{ }^{* * * *}=p<0.0001$. (D) Whole cell currents measured at holding potential ranging from $-100 \mathrm{mV}$ to $+80 \mathrm{mV}$ (in steps of $20 \mathrm{mV}$ ) in DAOY cells $+/-$ KO of MAP4K4 ( $n=13-16$ cells measured independently). (E) Quantification of the membrane potential of DAOY cells +/- KO of MAP4K4 and +/- HGF stimulation (20 ng/ml, $30 \mathrm{~min})$ and in normal [Cl-] or low [Cl-] (5 mM) in SFM medium. $\mathrm{N}=8-16$ cells measured independently. (F) U133P Affymetrix gene chip 
micro array analysis of CLIC1 mRNA expression levels in normal brain tissue and MB tumor samples. (G) Kaplan Meier survival analyses in four MB subgroups relative to CLIC1 mRNA expression levels.

\section{Figure 3}

PVR/CD155 promotes migration and invasion. (A) Quantification of PM association of PVR/CD155. Box plots display normalized z-score values extracted from all-group comparison of MS analysis. $\mathrm{N}=$ 4 independent experiments, mean \& SD, ${ }^{* *}=p<0.01$. (B) qrt-PCR analysis of PVR/CD155 mRNA expression in siCTL and siMAP4K4 -/+ HGF stimulation (30 min). (C) Surface expression analysis of PVR/CD155 by flow cytometry. Left: Histograms of PE intensity of DAOY cells with treatments as indicated. Right: bar diagram depicting average PE intensities relative to unstimulated siCTL cells. (D) SIA of siCTL, siCD155 or siCD276 transfected DAOY cells -/+ HGF stimulation. Left: Representative images of Hoechst-stained nuclei in inverted greyscale. Scale bar $=300 \mu \mathrm{m}$. Right Box plots display sum of distances of invasion ( $n=3$ independent experiments, ${ }^{*}=p<0.05,{ }^{* * * *}=p<0.0001$.). (E) SIA of siCTL or siCD155 transfected ONS-76 cells. Left: Representative images of Hoechst-stained nuclei in inverted greyscale. Scale bar $=300 \mu \mathrm{m}$. Right Box plots display sum of distances of invasion $(\mathrm{n}=3$ independent experiments. $\left.{ }^{*}=p<0.05\right)$. (F) Single cell motility analysis of siCTL and siCD155 transfected ONS-76 cells. Inverted grey-scale images are representative LA-EGFP fluorescence images of siRNA transfected DAOY cells seeded on collagen-I coated plates. Scale bar $=25 \mu \mathrm{m}$. Speed and morphological properties of cells were quantified in $5 \mathrm{~min}$ intervals for $300 \mathrm{~min}$ and are displayed over time (x/y plots) and averaged (box-dot plots). $\mathrm{N}=3$ independent experiments, mean \& SD, ${ }^{* * * *}=$ $p<0.0001$, ns $=$ not significant. (G) OCSC of siCTL or siCD155 ONS-76 cells implanted on cerebellar slices for 48h. Left: representative images of OCSCs. Red: Human nuclei, green: Calbindin (Purkinje cells), Scale bar: $400 \mu \mathrm{m}$. Right: Bar plots show volume and proliferation index of $\mathrm{n}=3$ spheroids ** $=$ $p<0.01$.

\section{Figure 4}

EndoA1 is required for migration and tissue invasion (A) Boyden chamber transwell migration assay using siRNA transfected DAOY cells. siRNAs as indicated. Significance is calculated from the comparison of each individual sample with the HGF-stimulated siCTL sample. $\mathrm{N}=3-4$ independent experiments, mean \& SD, ${ }^{*}=p<0.05,{ }^{* *}=p<0.01,{ }^{* * *}=p<0.001$. (B) Left: Representative images of DAOY spheroids \pm HGF $(20 \mathrm{ng} / \mathrm{ml})$ at endpoint. Inverted greyscale images of Hoechst-stained nuclei are shown. siRNA transfections as indicated. Scale bar $=300 \mu \mathrm{m}$. Right: Quantification of the invasion as sum of the distances from the center of spheroids shown in $\mathrm{A}$ (each dot represents 1 spheroid, $\mathrm{n}=$ 3 independent experiments, mean \& SD, ${ }^{* *}=p<0.01$, $\left.{ }^{* * * *}=p<0.0001\right)$. (C) Left: Representative images of ONS-76 spheroids. Inverted greyscale images of Hoechst-stained nuclei are shown. Scale bar $=300 \mu \mathrm{m}$. Right: Quantification of the invasion as sum of the distances as in $B,{ }^{*}=p<0.05$.. (D) Left: Representative images of DAOY spheroids grown from cells transfected with three siRNAs targeting Endo A1, A2 and A3 (TKD) \pm HGF $(20 \mathrm{ng} / \mathrm{ml})$. Inverted greyscale images of Hoechst-stained nuclei are shown. Scale bar $=300 \mu \mathrm{m}$. Right: Quantification of the invasion as sum of the distances as in $B,{ }^{* * * *}=p<0.0001$. Scale bar in all representative images $=300 \mu \mathrm{m}$. (E) Left: Maximum intensity 
projection of representative confocal sections of OCSC implanted with transfected ONS-76 spheroids $\pm 20 \mathrm{ng} / \mathrm{ml}$ HGF 48h after implantation. Cyan: Lifeact-EGFP; Magenta: calbindin (Purkinje cells). Right: Quantification of tumor cell areas and perimeters. ( $n=3$, technical replicates, mean \& SEM). Scale bar $=400 \mu \mathrm{m} . \mathrm{a}$ and $\mathrm{b}$ are higher magnification images of ONS-76 tumor cells at the invasion front (actin cytoskeleton of tumor cell in cyan, Purkinje cells in magenta).

\section{Figure 5}

Depletion of EndoA1 or MAP4K4 causes altered CIP4 localization (A) Quantification of PM association of regulators of FEME priming. Box plots display normalized z-score values extracted from all-group comparison of MS analysis. $\mathrm{N}=4$ independent experiments, mean $\& \mathrm{SD},{ }^{*}=\mathrm{p}<0.05,{ }^{* * * *}=$ $p<0.0001$. (B) Confocal microscopy analysis of CIP4 expression in siCTL, siMAP4K4 or siEndoA1 transfected ONS-76 cells. Magenta: LA-EGFP (F-actin), blue: Hoechst (DNA). (C) Confocal microscopy analysis of CIP4 expression in siCTL and EndoA1 transfected cells relative to F-actin. (D) Model for MAP4K4 regulation of the fast Endophilin mediated endocytosis. Under control conditions, during priming stages (1 and 2): CIP4 is recruited to the plasma membrane (PM). PM-associated CIP4 recruits EndoA1 to the plasma membrane. After receptor activation (3), endophilins initiate FEME and promote the internalization of the carrier and trafficking towards early endosomes. siRNA depletion of EndoA1 induce an accumulation of CIP4 at the PM during the priming stage. MAP4K4 could either promote priming complex disassembly or reduces EndoA1 recruitment to the PM and lead to CIP4 accumulation to the PM.

\section{Graphical abstract:}

C-MET activation upon HGF stimulation induces C-MET internalization and induces downstream MAP4K4 activity. (1) Activation of the kinase MAP4K4 is required for the maintenance and increased surface presentation of CD155. CD155 expression is required for MB cell migration, invasion and dissemination. (2) MAP4K4 activity contributes to the maintenance of MB cell membrane depolarization by regulating the surface localization of several ion channels and transporters. (3) MAP4K4 activity promotes FEME priming steps by regulating the PM-proximal localization of FEME effector CIP4 and potentially FBP17 and CIN85. The FEME effector endophilin A is necessary for MB cell migration, invasion and dissemination.

\section{Supplementary figures}

\section{Figure S1}

Validation of gene depletion efficiencies. (A) Immunoblot analysis of MAP4K4 expression in sgCTL and sgMAP4K4 DAOY cells used in PM-associated proteome analysis. Treatments as indicated (HGF $20 \mathrm{ng} / \mathrm{ml}, 30 \mathrm{~min}$ ). (B) Relative mRNA expression of a selection of proteins with significantly altered PM association. $\mathrm{N}=3$ independent experiments. , mean \& SD, ${ }^{*}=\mathrm{p}<0.05,{ }^{* *}=\mathrm{p}<0.01,{ }^{* * * *}=\mathrm{p}<0.0001$. 
(C) Relative mRNA expression analysis for $C D 155$ and $C D 276$ with siRNAs used. $\mathrm{N}=3$ independent experiments, mean \& SD, ${ }^{*}=p<0.05,{ }^{* *}=p<0.01,{ }^{* * * *}=p<0.0001$.

\section{Figure S2}

MAP4K4 promotes HGF-induced internalization of solute carriers and transporters. (A) Quantification of the cell-surface association of solute carriers and transporters involved in chemotherapy sensitivity and resistance. Relative z-Score value extracted from all-group comparison. $\left(\mathrm{n}=4\right.$ independent experiments, Mean \& SD, $\left.{ }^{*} \mathrm{p}<0.05,{ }^{* *} p<0.01,{ }^{* * *} \mathrm{p}<0.001\right)$. (B) Dose-response curve and corresponding IC50 values of Lomustine and Etoposide after 48 hours treatment under standard culture conditions. (C) Dose-response curve and corresponding IC50 values of Lomustine and Etoposide after 48 hours treatment +/- HGF stimulation under low-serum culture conditions. $(n \geq 3$ technical replicates, mean + SEM)

\section{Figure S3}

Proportion of plasma membrane and transmembrane predicted transporters (A) Pie chart representing the proportion of PM-associated proteins (red) in all proteins identified in MS analysis. Proteins annotated as transporters using Protein Atlas are highlight in dark red. (B) Pie chart representing the proportion of proteins with predicted transmembrane domains (red). Proteins annotated as transporters using Protein Atlas are highlight in dark red. (C) Selection of proteins with predicted transmembrane domains with an implication in ion transport. Potential ions involved are indicated. Normalized z-score values extracted from all-group comparison of MS analysis are depicted. Fold change and $\mathrm{p}$-values extracted from each two-group analysis are shown. $\mathrm{N}=4$ independent experiments. Green: upregulated, red: downregulated, black: unchanged.

\section{Figure S4}

CD155 depletion prevents tissue invasion ex vivo. Confocal microscopy analysis of OCSCs two days after implantation of spheroid of DOAY cells transfected with indicated siRNAs. Cyan: Human nuclei, Magenta: Calbindin (Purkinje cells). Tumor cell area is indicated with dashed line in inverted gray-scale confocal images. Scale bar $=400 \mu \mathrm{m}$.

\section{Figure S5}

SH3GL2 is highly expressed in SHH MB tumors and correlates with MAP4K4 expression. (A) MAP4K4 co-expressed genes from 763 primary medulloblastoma samples were used to interrogate KEGG pathway database. (B) Top 20 genes with a positive or negative correlative expression score with MAP4K4 in primary medulloblastoma samples. (C) Two gene analyses of SH3GL2, MAP4K4 and CLTC expression across the four MB subgroups. Correlation coefficient $(r)$ and $p$-Value was calculated 
from all four subgroups combined. (D) Expression of MAP4K4, SH3GL1, SH3GL2 and SH3GL3 at the RNA and proteomic level in 218 pediatric brain tumors across seven histologic types. Copy number variation (CNV) and mutations are represented if available. The data were obtained from the R2: Genomics Analysis and Visualization platform (http://r2.amc.nl) and CPTAC data portal (http://pbt.cptac-data-view.org/).

\section{Figure S6}

Validation experiments on the implication of endocytosis on MB tumor progression (A) Schematic representation of endocytic pathways in mammalian cells. (B) List of target genes used for siRNA screening and corresponding endocytic pathways. Pathways with dynamin-independent vesicle closure and abscission are shown in turquoise. (C) Efficacy analysis of siRNAs used in transwell migration screen by qRT-PCR with the Dharmacon transfection reagent. RNA extraction occurred at endpoint of Boyden chamber assay. ( $N=2$ to 9 independent experiments, Mean $+S D$ ). (D) Relative quantification of cell proliferation. ( $N=2$ independent experiments, Mean + SEM). (E) Efficacy analysis of siRNAs used in all other experiments by qRT-PCR with the Invitrogen transfection reagent. RNA extraction occurred at endpoint of SIA. ( $N=6$ independent experiments, Mean $+S D$, $\left.{ }^{* * * *} p<0.0001\right)$. (F) Immunoblot analysis of siRNA-mediated Endophilin-A1 depletion in ONS-76 cells and quantification $(\mathrm{N}=3$ independent experiments, Mean $+\mathrm{SD}) .{ }^{* *} \mathrm{p}<0.01, \mathrm{~ns}=$ not significant. (G) Left: Confocal microscopy analysis of OCSCs two days after implantation of spheroid of DOAY cells transfected with indicated siRNAs. Cyan: LA-EGFP, Magenta: Calbindin (Purkinje cells), yellow: Human nuclei. Tumor cell area is indicated with dashed line in inverted gray-scale confocal images. Scale bar $=400 \mu \mathrm{m}$. Right: proliferation index of $n=3$ spheroids.

\section{Figure S7}

EndoA1- and MAP4K4-mediated intracellular vesicular content regulation (A) Confocal microscopy analysis of the early endosome marker EEA1. Left: Maximum intensity projection of siCTL, siEndoA1 and siMAP4K4 transfected DAOY cells. Blue: Nucleic acids, Orange: EEA1. Scale bar $=50$ $\mu \mathrm{m}$. Right: Quantification of the EEA1 positive puncta per cell. (B) Confocal microscopy analysis of the late endosome marker LAMP1. Left: Maximum intensity projection of siCTL, siEndoA1 and siMAP4K4 transfected DAOY cells. Blue: Nucleic acids, Green: LAMP1. Scale bar $=50 \mu \mathrm{m}$. Right: Quantification of the LAMP1 positive puncta per cell. For each condition, EEA1 and LAMP1 pictures represent the same field of view. $\mathrm{N}=4$ technical replicates Min to Max, ${ }^{*} p<0.05,{ }^{* *} p<0.01,{ }^{* * *} p<0.001,{ }^{* * * *} p<$ $0.0001, \mathrm{~ns}=$ not significant.

\section{Supplementary files}

Table 1 | Surface Proteome Transmembrane prediction TMHMM filtering

Excel File: Table 1_SurfaceProteome_TransMembraneAnnotation-TMHMM-Filtering_Capdeville2021 
Table 2 | Surface Proteome Plasma membrane annotation filtering

Excel File: Table 2_SurfaceProteome_PlasmaMembraneAssociation_Capdeville2021

Table 3 | Significant fold change for Transmembrane predicted proteins

Excel File: Table3_Significant_FoldChange_TransMembraneAnnotation_Capdeville2021

Table 4 | Significant fold change for Plasma membrane annotated proteins

Excel File: Table4_Significant_FoldChange_PlasmaMembraneAssociation_Capdeville2021

\section{Supplementary materials}

Table S1 | List of siRNAs used in this study

\begin{tabular}{|c|c|c|c|}
\hline \multicolumn{4}{|c|}{ siRNAs } \\
\hline Target gene & NCBI Gene ID & Identifier & Company \\
\hline CTL & - & 4390643 & \multirow{19}{*}{$\begin{array}{c}\text { Thermo Fisher Scientific } \\
- \\
\text { Silencer® Select }\end{array}$} \\
\hline CD155 & 5817 & s11602 & \\
\hline CD276 & 80381 & s37290 & \\
\hline \multirow{2}{*}{ DNM1 } & \multirow{2}{*}{1759} & s146 & \\
\hline & & s145 & \\
\hline DNM2 & 1785 & s4213 & \\
\hline CLTC & 1213 & s475 & \\
\hline \multirow{2}{*}{ LDLR } & \multirow{2}{*}{3949} & s224006 & \\
\hline & & s4 & \\
\hline RAC1 & 5879 & s11713 & \\
\hline CTBP1 & 1487 & s3699 & \\
\hline CAV1 & 857 & s2446 & \\
\hline \multirow{2}{*}{ SH3GL2 } & \multirow{2}{*}{6456} & s12800 & \\
\hline & & $\mathrm{s} 12801$ & \\
\hline \multirow{2}{*}{ SH3GL1 } & \multirow{2}{*}{6455} & s12798 & \\
\hline & & s12796 & \\
\hline \multirow{2}{*}{ SH3GL3 } & \multirow{2}{*}{6457} & s227202 & \\
\hline & & s12802 & \\
\hline FLOT1 & 10211 & s19914 & \\
\hline
\end{tabular}




\begin{tabular}{|c|c|c|c|}
\hline & & s19913 & \\
\hline \multirow{2}{*}{ FLOT2 } & \multirow{2}{*}{2319} & s5284 & \\
\hline & & s5286 & \\
\hline \multirow{2}{*}{ CDC42 } & \multirow{2}{*}{998} & s2765 & \\
\hline & & s2766 & \\
\hline \multirow{2}{*}{ GRAF1 } & \multirow{2}{*}{23092} & s23014 & \\
\hline & & s23092 & \\
\hline ARF6 & 382 & s1565 & \\
\hline RHOA & 387 & s758 & \\
\hline MAP4K4 & 9448 & D-003971-05-0002 & Dharmacon \\
\hline
\end{tabular}

Table S2 | Custom qPCR primers used in this study

\begin{tabular}{|c|c|c|}
\hline \multicolumn{3}{|c|}{ Sybr Green qPCR primers } \\
\hline \multicolumn{2}{|c|}{ Target gene } & Sequence \\
\hline \multirow{2}{*}{$18 S$} & Fw & GGATGTAAAGGATGGAAAATACA \\
\hline & Rv & TCCAGGTCTTCACGGAGCTTGTT \\
\hline \multirow{2}{*}{ CD155 } & $\mathrm{Fw}$ & CAGTGAGCACTCAGGTACAGA \\
\hline & $\mathrm{Rv}$ & TCACCTTGTGCCCTCTGTCT \\
\hline \multirow{2}{*}{ CD276 } & Fw & ACAGGAAGATGCTGCGTCG \\
\hline & $\mathrm{Rv}$ & AGGGCTCCTGTGAGGCA \\
\hline \multirow{2}{*}{ CLIC1 } & Fw & GGTGGGGAGGCCTAGTTTG \\
\hline & $\mathrm{Rv}$ & CAGGGACTCTCTCCCCTAGAC \\
\hline \multirow{2}{*}{ SLC29A1 } & Fw & СТTACССАТСАТGСАСССТGТ \\
\hline & $\mathrm{Rv}$ & AGAGAGCCTCTGAAGGCACC \\
\hline \multirow{2}{*}{ LRRC8A } & Fw & GAGCAAAAGGAATGCCAGGA \\
\hline & $\mathrm{Rv}$ & TCATGGTTCAACCCCAAGGA \\
\hline \multirow{2}{*}{$\mathrm{ABCC} 1$} & Fw & TGCATCGTTCTGTTTGCTGC \\
\hline & $\mathrm{Rv}$ & TCAAGTACGTGGTGACCTGC \\
\hline \multirow{2}{*}{ MAP4K4 } & Fw & GTTACACTAATGCGCACCAC \\
\hline & $\mathrm{Rv}$ & GTACTTGCCACCAGTCTGCT \\
\hline \multirow{2}{*}{ GAPDH } & Fw & GACAGTCAGCCGCATCTTCT \\
\hline & $\mathrm{Rv}$ & TTAAAAGCAGCCCTGGTGAC \\
\hline \multirow{2}{*}{ DNM1 } & Fw & TCCATGGCATTAGAACGGGG \\
\hline & $\mathrm{Rv}$ & TCCACACACTTGAGACACGG \\
\hline DNM2 & Fw & CAACACGGAGCAGAGAAACG \\
\hline
\end{tabular}




\begin{tabular}{|c|c|c|}
\hline & $\mathrm{Rv}$ & CTTCCAGCTGTCCACGTCTT \\
\hline \multirow{2}{*}{ CLTC } & Fw & CAATTTCAGCAGACAGCGCC \\
\hline & Rv & TCTGAAGAGTTTTCCCAGATTCTTT \\
\hline \multirow{2}{*}{ LDLR } & Fw & ACCACAGAGGATGAGGTCCA \\
\hline & Rv & TGACCATCTGTCTCGAGGGG \\
\hline \multirow{2}{*}{ RAC1 } & Fw & AAACCGGTGAATCTGGGCTT \\
\hline & Rv & AGAACACATCTGTTTGCGGA \\
\hline \multirow{2}{*}{ CTBP1 } & Fw & GGCCTGCCGCTTGTAAGAC \\
\hline & $\mathrm{Rv}$ & TGTCTCGAGCCAAAGTGCTC \\
\hline \multirow{2}{*}{ CAV1 } & Fw & TCTTCCAACACGTAGCTGCC \\
\hline & $\mathrm{Rv}$ & CGGTGTAGAGATGTCCCTGCG \\
\hline \multirow{2}{*}{ SH3GL2 } & Fw & AGTTCCATAAAGCCACTCAGAAAG \\
\hline & $\mathrm{Rv}$ & CCTGCTGGTGACATCCACTT \\
\hline \multirow{2}{*}{ SH3GL1 } & Fw & AGAGGTGAAGGACTCCCTGG \\
\hline & $\mathrm{Rv}$ & AGGTGGTGCTGGATCTCCTT \\
\hline \multirow{2}{*}{ SH3GL3 } & Fw & TCTTCAGCCAAATCCAGAGCTA \\
\hline & $\mathrm{Rv}$ & GCGGGTATCCTGTGGTCTTC \\
\hline \multirow{2}{*}{ FLOT1 } & Fw & GGCATTGCCCAGGAGATCTAT \\
\hline & $\mathrm{Rv}$ & ACCACACTGATGCCCATGTT \\
\hline \multirow{2}{*}{ FLOT2 } & Fw & ATGACCATCCTGTGTCGCTG \\
\hline & $\mathrm{Rv}$ & ATCTTCACCTGTGCAACCCC \\
\hline \multirow{2}{*}{ CDC42 } & Fw & CGACCGCTGAGTTATCCACA \\
\hline & Rv & TCTCAGGCACCCACTTTTCT \\
\hline \multirow{2}{*}{ GRAF1 } & Fw & TAGTCCGCACTTCCGAGAGA \\
\hline & $\mathrm{Rv}$ & CGCTTCGCTGAAGACAAATTCTTG \\
\hline \multirow{2}{*}{ ARF6 } & Fw & GGAAGTGAGGCGGTTTCCTC \\
\hline & Rv & GGTGCGCTGCCTCCAG \\
\hline \multirow{2}{*}{ RHOA } & Fw & AGCCAAGATGAAGCAGGAGC \\
\hline & $\mathrm{Rv}$ & TACCCAAAAGCGCCAATCCT \\
\hline
\end{tabular}

Table S3 | List of antibodies used in this study

\begin{tabular}{|c|c|c|c|}
\hline \multicolumn{5}{|c|}{ Antibodies } \\
\hline Antibody name & Identifier & Company & Dilution \\
\hline Anti-Calbindin & ab108404 & Abcam & $1: 1000$ (IF) \\
\hline Anti-human nuclei & MAB4383 & Millipore & $1: 250$ (IF) \\
\hline
\end{tabular}




\begin{tabular}{|c|c|c|c|}
\hline Anti-MAP4K4 & $a b 80418$ & Abcam & $1: 2000(\mathrm{WB})$ \\
\hline Anti-CIP4 & sc-135868 & $\begin{array}{c}\text { Santa Cruz } \\
\text { Biotechnology }\end{array}$ & $1: 250$ (IF) \\
\hline Anti-SH3GL2 & $a b 150431$ & Abcam & 1:1000 (WB) \\
\hline Anti-EEA1 & 2411 & $\begin{array}{l}\text { Cell Signaling } \\
\text { Technology }\end{array}$ & $1: 200$ (IF) \\
\hline Anti-LAMP1 & 555798 & BD Biosciences & $1: 250$ (IF) \\
\hline Anti-GAPDH & $2118 s$ & $\begin{array}{l}\text { Cell signaling } \\
\text { technologies }\end{array}$ & 1:2000 (WB) \\
\hline PE-conjugated anti-CD155 & 337610 & BioLegend & $1: 300$ (FACS) \\
\hline $\begin{array}{l}\text { PE-conjugated IgG1, } \mathrm{k} \\
\text { Isotype Ctrl }\end{array}$ & 400114 & BioLegend & $1: 300$ (FACS) \\
\hline $\begin{array}{c}\text { Cy3-conjugated Donkey anti- } \\
\text { Rabbit IgG }\end{array}$ & $711-165-152$ & $\begin{array}{c}\text { Jackson } \\
\text { ImmunoResearch }\end{array}$ & $1: 250$ (IF) \\
\hline $\begin{array}{c}\text { Goat anti-Mouse lgG } \\
\text { Secondary Antibody, Alexa } \\
\text { Fluor } 405\end{array}$ & A31553 & Thermo Fisher Scientific & $1: 200$ (IF) \\
\hline $\begin{array}{c}\text { Cy5-conjugated Donkey anti- } \\
\text { Mouse IgG }\end{array}$ & $715-175-150$ & $\begin{array}{c}\text { Jackson } \\
\text { ImmunoResearch }\end{array}$ & $1: 250$ (IF) \\
\hline $\begin{array}{c}\text { Goat anti-Rabbit IgG Cross- } \\
\text { Adsorbed Secondary } \\
\text { Antibody, Alexa Fluor } 568\end{array}$ & A-11011 & Thermo Fisher Scientific & 1:200 (IF) \\
\hline $\begin{array}{l}\text { Goat anti-Mouse IgG Cross- } \\
\text { Adsorbed Secondary } \\
\text { Antibody, Alexa Fluor } 488\end{array}$ & A-11001 & Thermo Fisher Scientific & $1: 400$ (IF) \\
\hline
\end{tabular}



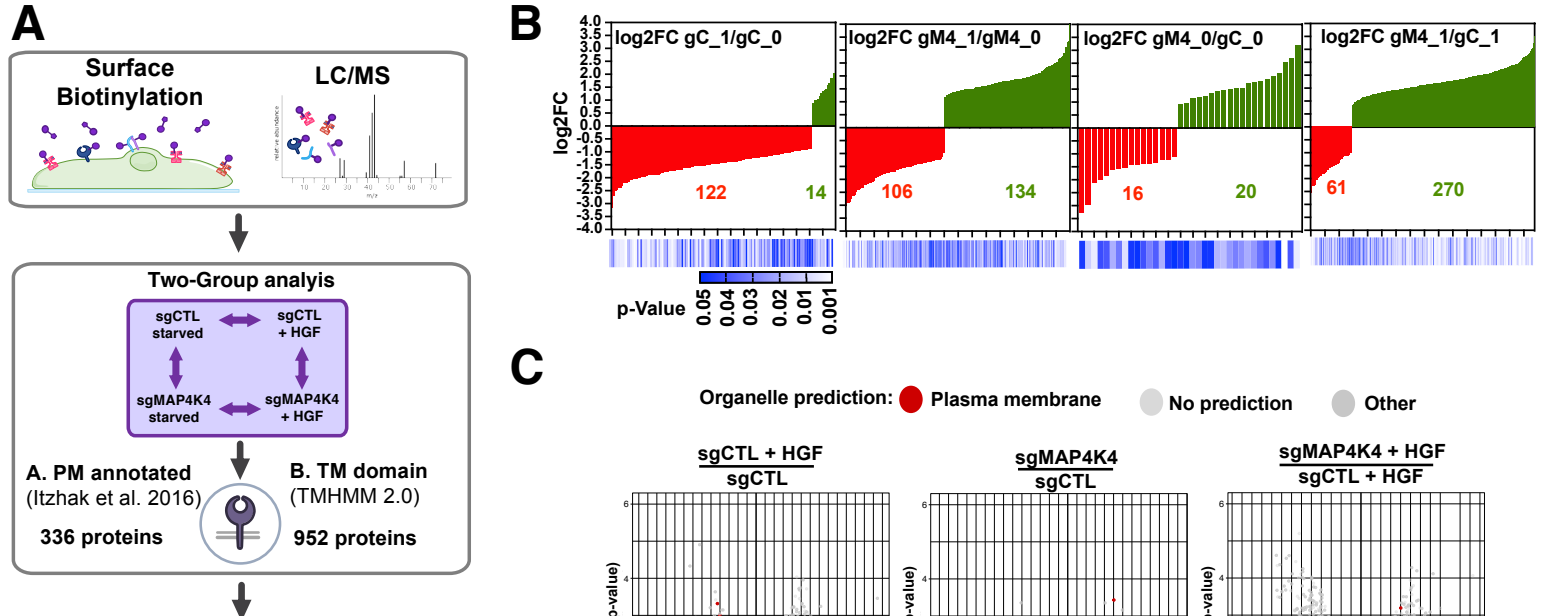

C

Pathway enrichment analysis on significantly altered proteins
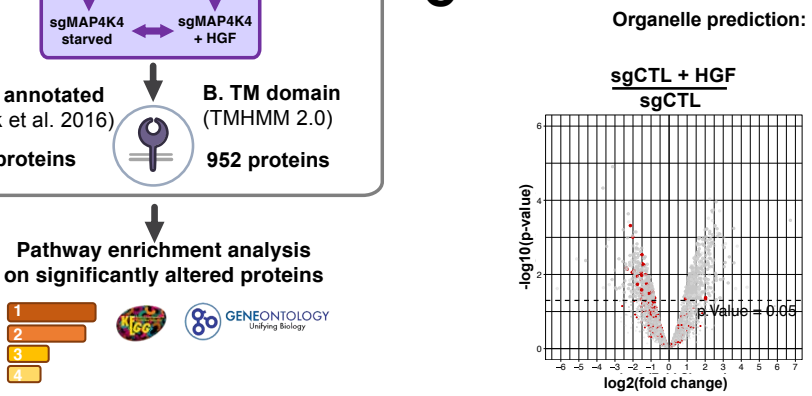

Plasma membrane

No prediction Other
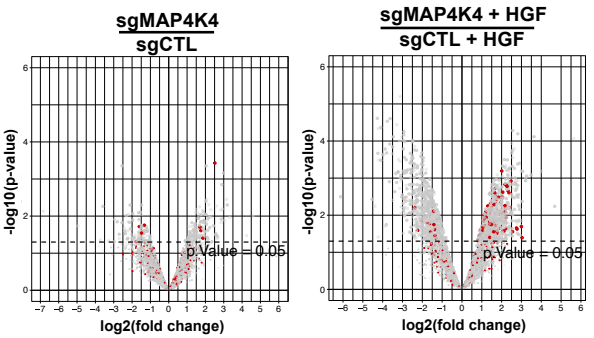

D

E Fold change

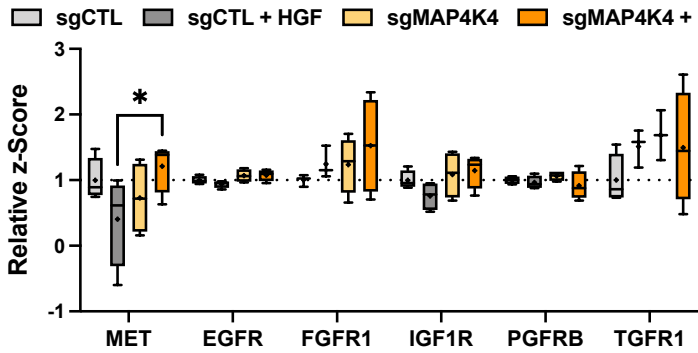

$\mathbf{F}$

Effect of Effect of Effect of HGF: MAP4K4 KO: MAP4K4 KO:

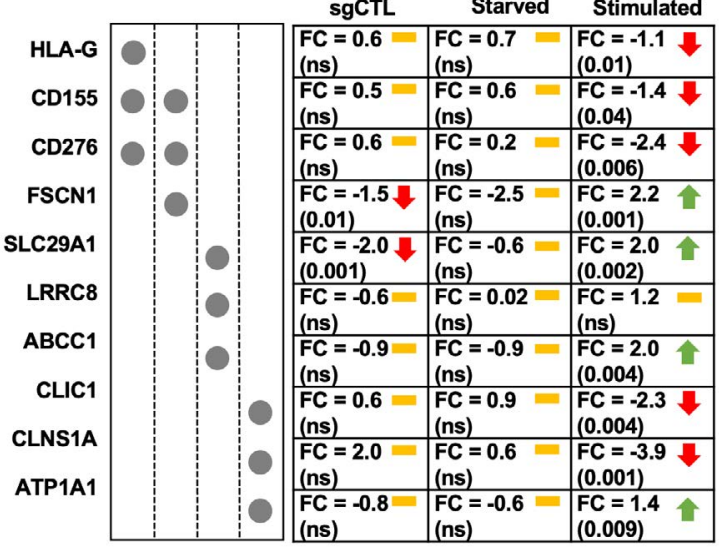

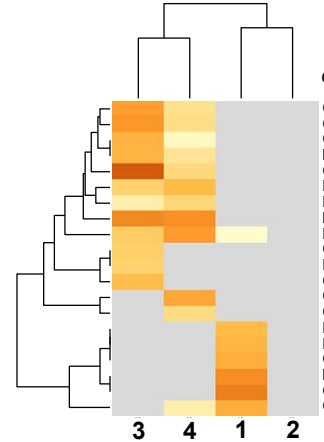

\begin{tabular}{|l}
$-\log 10(P)$ \\
$024610 \quad 20$
\end{tabular}

GO:0042110: $T$ cell activation GO:0046718: viral entry into host cell effector proces (c0.003134: GO:0031341: regulation of cell killing

Or clathrin-mediated endocytosis .

R-HSA-202733: Cell surface interactions at the vascular wall GO:0034113: heterotypic cell-cell adhesion R-HSA-1280215: Cytokine Signaling in Immune system GO:0009617: response to bacterium GO:1903670: regulation of sprouting angiogenesis GO:1990778: protein localization to cell periphery R-HSA-425407: SLC-mediated transmembrane transport RA

ion-dependent exocytosis  exocytosis

$\begin{array}{lll}3 & 4 & 1\end{array}$

\section{Fold change}

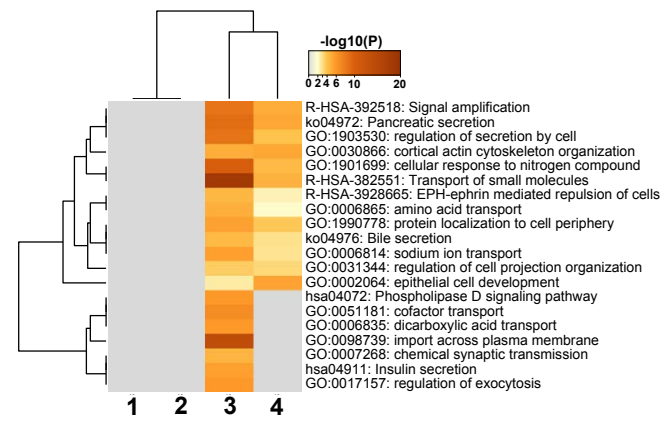

1: sgCTL + HGF vs sgCTL

2: sgMAP4K4 vs sgCTL

3: sgMAP4K4 + HGF vs sgCTL + HGF

4: sgMAP4K4 + HGF vs sgMAP4K4

1. Immune evasion

2. Cell migration

3. Chemotherapy sensitivity

4. Ion homeostasis 
A $\quad$ CLIC1

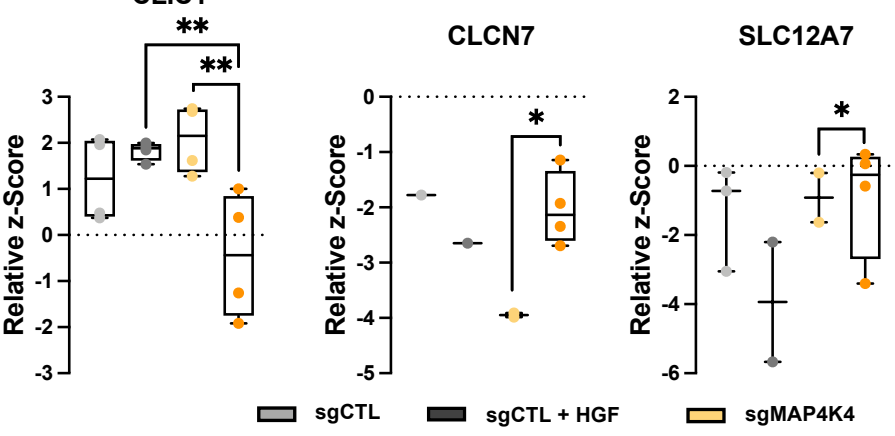

ATP1B3

TMEM165

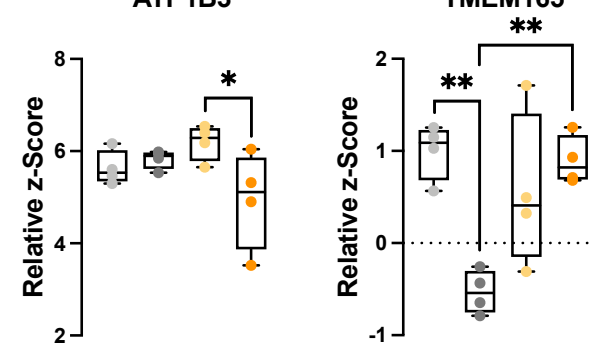

B

sgMAP4K4 + HGF/ sgCTL + HGF

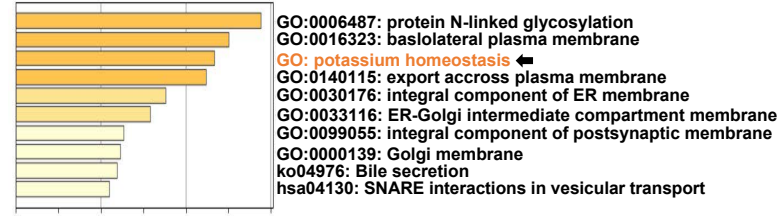

GO:0016323: baslolateral plasma membran

hsa04130: SNARE interactions in vesicular transport

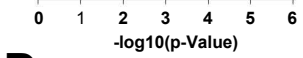

D

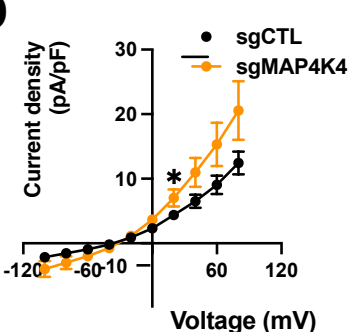

$\mathbf{F}$
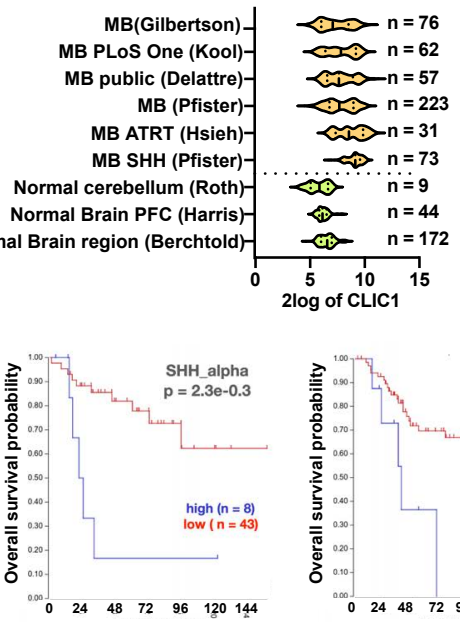

Follow up in months

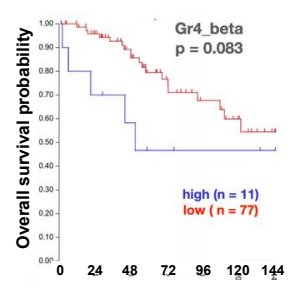

Follow up in months
$\mathbf{E}$

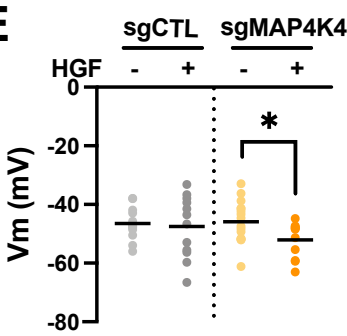

C
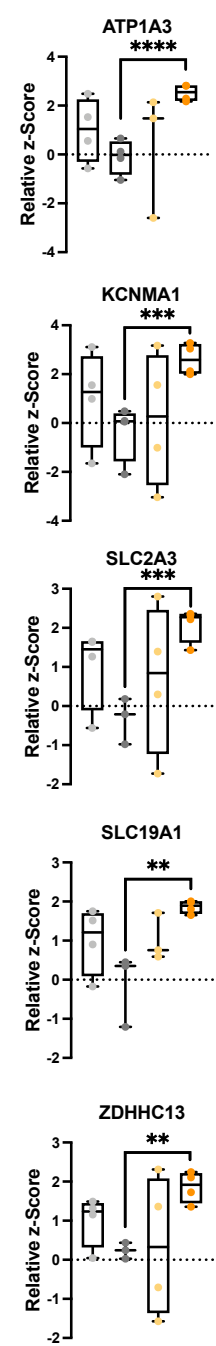

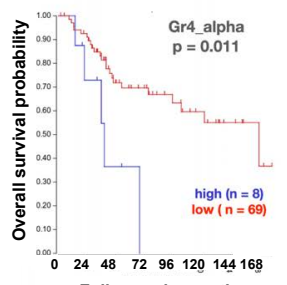

Follow up in months

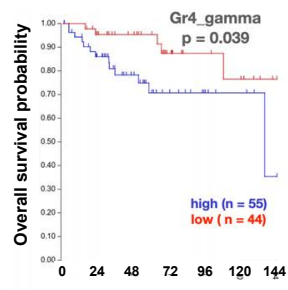

sgMAP4K4 + HGF

GO:0055075 Potassium ion homeostasis
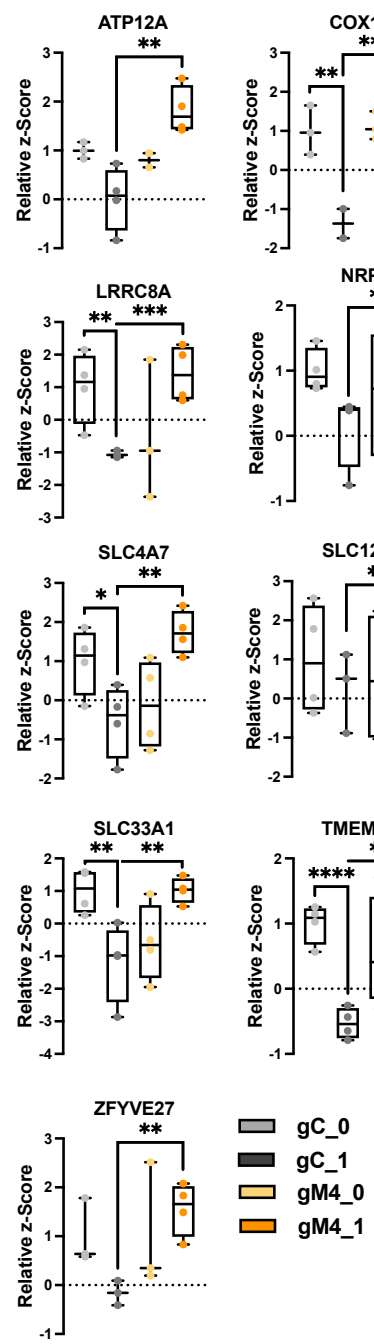
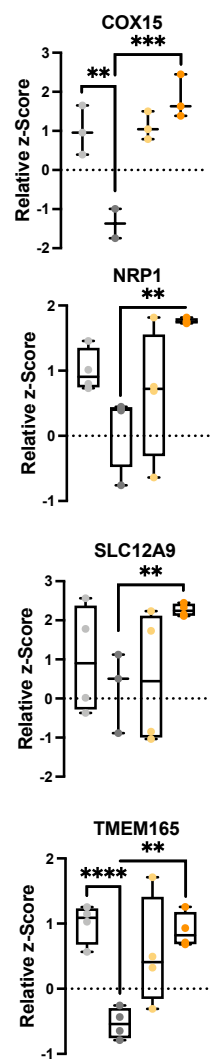

Fig. 2 


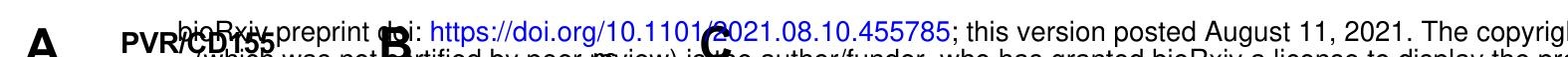
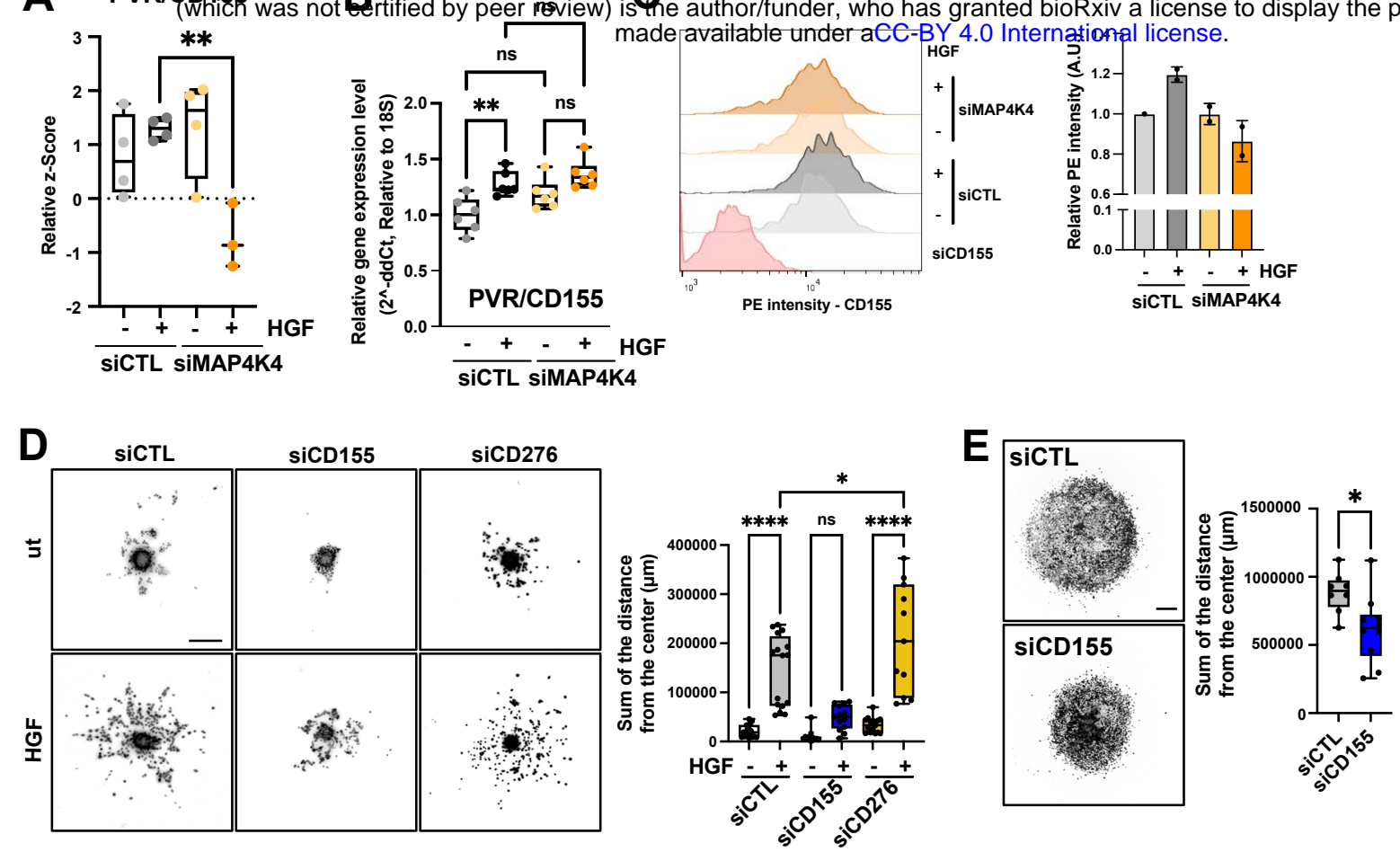

$\mathbf{F}$
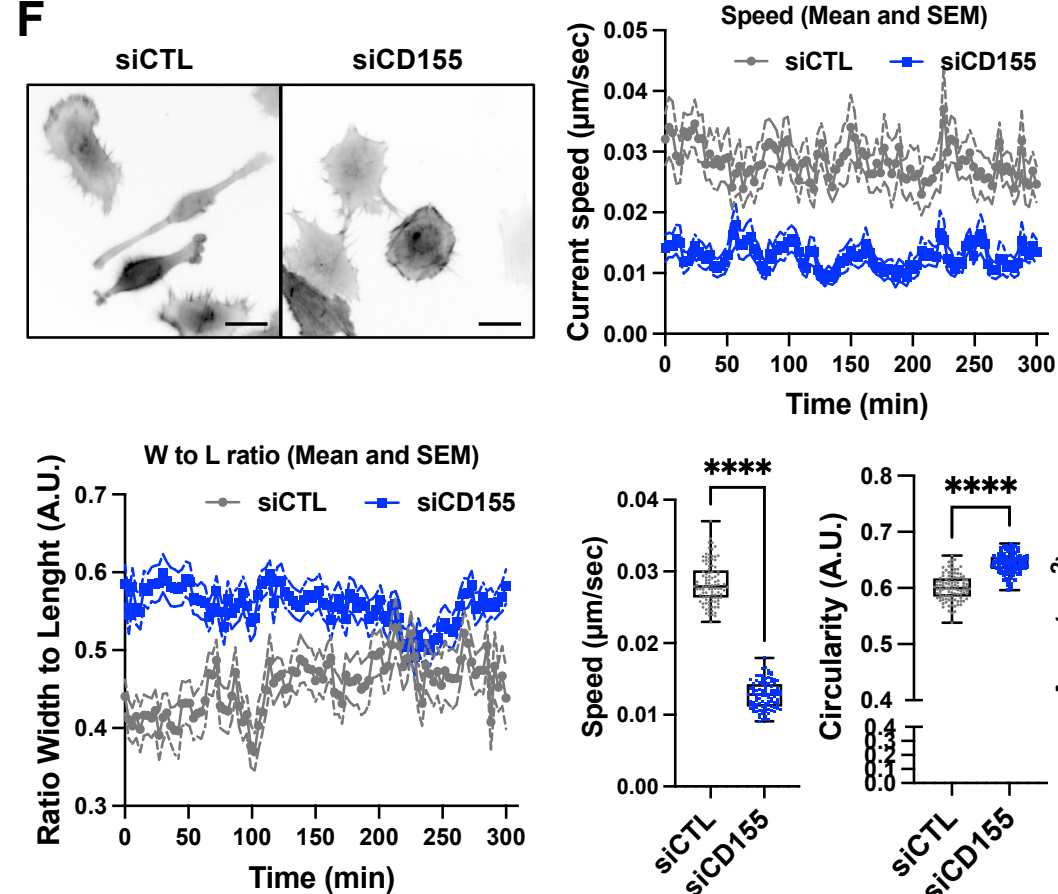

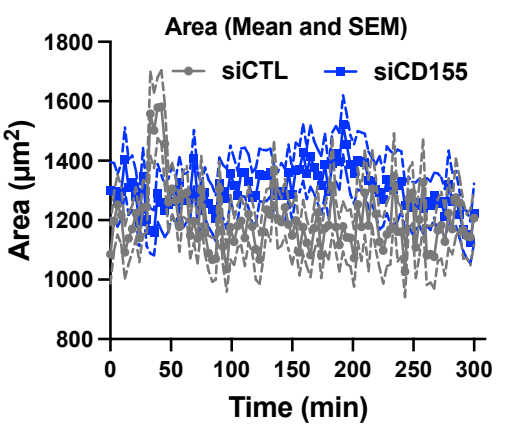

G

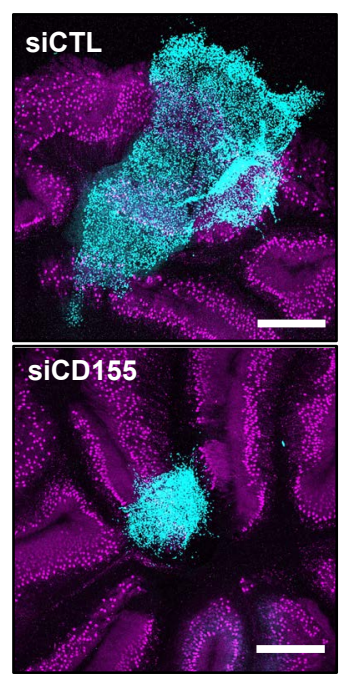

Human nucle

Calbindin
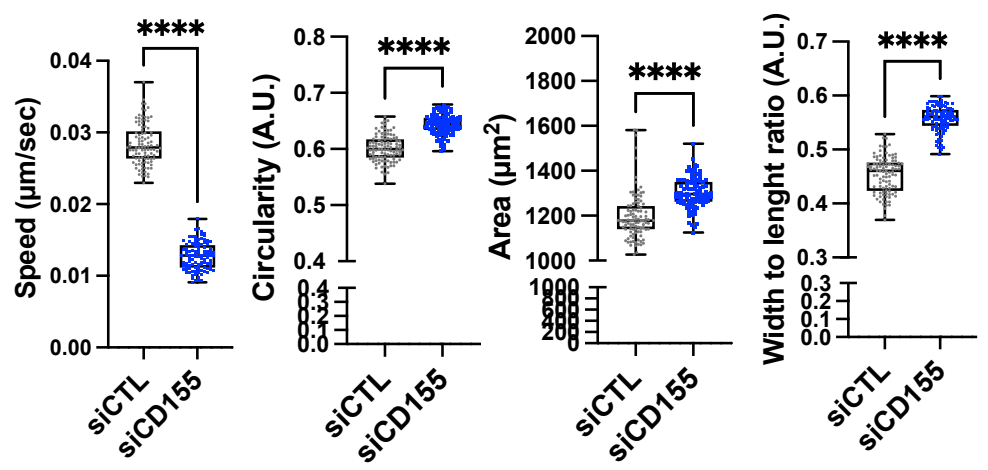

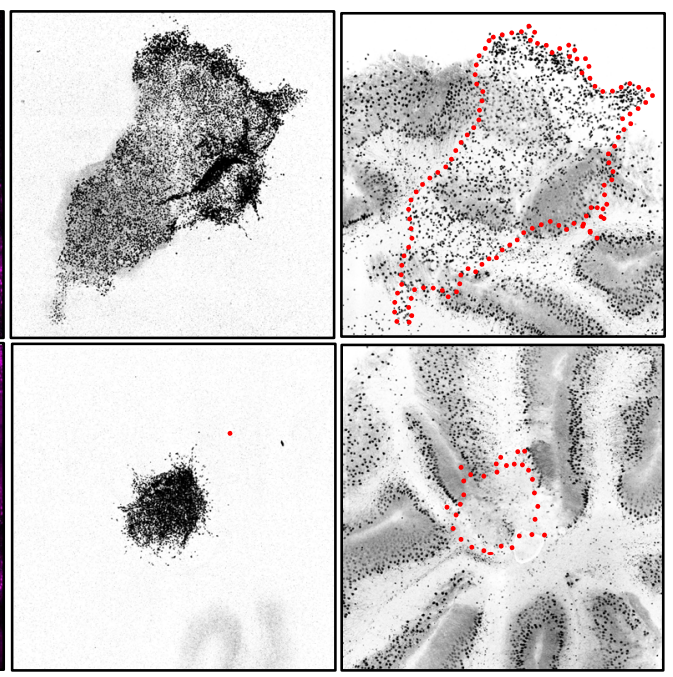

Human nuclei

Calbindin/EdU

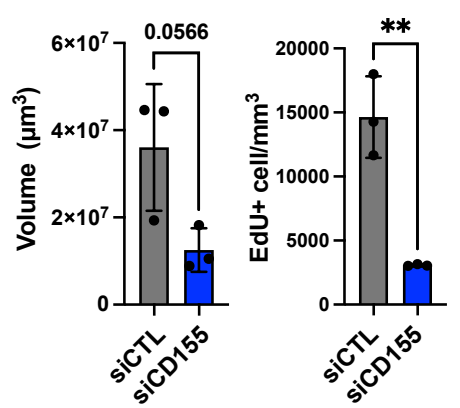

Fig. 3 
